\title{
Management of antiplatelet therapy in patients undergoing neuroendovascular procedures
}

\author{
Keri S. Kim, PharmD, MS CTS, ${ }^{1}$ Justin F. Fraser, MD, ${ }^{2}$ Stephen Grupke, MD, ${ }^{3}$ and \\ Aaron M. Cook, PharmD ${ }^{4,5}$
}

\begin{abstract}
1Department of Pharmacy Practice, University of Illinois Medical Center at Chicago, Illinois; 2Departments of Neurological Surgery, Neurology, Radiology, and Anatomy and Neurobiology, Center for Advanced Translational Stroke Science; ${ }^{3}$ Department of Neurological Surgery, UK HealthCare; “UK HealthCare; and ${ }^{5}$ University of Kentucky College of Pharmacy, Lexington, Kentucky
\end{abstract}

\begin{abstract}
Neuroendovascular techniques for treating cerebral aneurysms and other cerebrovascular pathology are increasingly becoming the standard of care. Intraluminal stents, aneurysm coils, and other flow diversion devices typically require concomitant antiplatelet therapy to reduce thromboembolic complications. The variability inherent with the pharmacodynamic response to common antiplatelet agents such as aspirin and clopidogrel complicates optimal selection of antiplatelet agents by clinicians. This review serves to discuss the literature related to antiplatelet use in neuroendovascular procedures and provides recommendations for clinicians on how to approach patients with variable response to antiplatelet agents, particularly clopidogrel.
\end{abstract}

https://thejns.org/doi/abs/10.3171/2017.5.JNS162307

KEY WORDS aneurysm; clopidogrel; intracranial stenosis; platelet reactivity; vascular disorders

$\mathrm{E}$ NDOVASCULAR techniques are increasingly becoming the standard for the treatment of intracranial aneurysms and other cerebrovascular pathology. Stent and flow diversion techniques are often used, requiring peri- and postprocedural medical therapy, in particular antiplatelet medications, to minimize complications. Adequately trained operators and appropriate facilities are still relatively limited across the US; thus, many of these procedures are primarily performed at regional referral centers. The intent of this review is to introduce clinicians to the role of antiplatelet pharmacotherapy in preventing complications during and after various types of neuroendovascular interventions. The current dependence on complicated antiplatelet regimens and the variability in patient-specific response has led to the increased use of platelet function testing to determine the optimal dose and choice of medication. The role of platelet function testing and the use of agents for thromboembolic rescue in this paradigm will also be reviewed.

\section{Neuroendovascular Therapeutics}

Coil embolization, most commonly known for its role in the treatment of cerebral aneurysms, is the process by which a series of small (typically $0.010-0.018$-inch) platinum coils are passed through a catheter into the lumen of an aneurysm to occlude it, thereby preventing rupture of the aneurysm. These coils are procoagulant by nature, with a high surface area, leading to early thrombosis and ultimately formation of vascular collagenous scar tissue. ${ }^{10,11}$ The use of antiplatelet therapy in the periprocedural period is common due to the significant risk of thromboembolic events, as high as $12.5 \%$ in 1 large study, although this must be weighed among other hemorrhagic risk factors, especially in the setting of a ruptured aneurysm. ${ }^{52}$ While stent-assisted coiling has allowed for the endovascular treatment of lesions once deemed "uncoilable," the stents pose a risk for thrombosis through perturbation of the endothelium and release of procoagulant factors into the blood, as well as by providing a lattice on which thrombi may form. Mocco et al. demonstrated a $4 \%$ risk of thromboembolic events with stent-assisted coiling; however, $1 \%$ were intraprocedural and immediately corrected, while all others were associated with interruption of periprocedural dual antiplatelet therapy (DAT). ${ }^{42}$

Aside from being a structural adjunct, some intracranial stents have been developed to treat aneurysms alone by way

ABBREVIATIONS ACS = acute coronary syndrome; $A R U$ = aspirin reaction unit; $C Y P=$ cytochrome P450; DAT = dual antiplatelet therapy; DWI = diffusion-weighted imaging; GRAVITAS = Gauging Responsiveness with a VerifyNow P2Y12 assay: Impact on Thrombosis and Safety; HTPR = high on-treatment platelet reactivity; LTA = light/optical transmission aggregometry; LTPR = low on-treatment platelet reactivity; $\mathrm{PCI}=$ percutaneous coronary intervention; PED = Pipeline embolization device; PRU = P2Y12 reaction unit.

SUBMITTED September 5, 2016. ACCEPTED May 15, 2017.

INCLUDE WHEN CITING Published online December 1, 2017; DOI: 10.3171/2017.5.JNS162307. 
of diverting blood flow away from the body of the lesion and permitting natural thrombosis and inflammatory pathways to allow the aneurysm to resolve over time. Thrombosis rates with the Pipeline embolization device (PED; ev3 Neurovascular) have tended to be higher than non-flowdiverting stents, with thrombosis rates ranging from $8 \%$ to $12 \%$, even when combined with DAT. ${ }^{14,32,38}$ The 6-month incidence of in-stent stenosis has reportedly ranged from $3.5 \%$ to $16 \% .^{12}$ Because of these issues, significant interest has developed in the neurointerventional community regarding the optimization of antiplatelet therapy.

\section{Platelet Function}

Platelets contribute to the development of both hemostasis and thrombosis as part of the normal physiological response. In clinical practice, extreme conditions causing increased or decreased platelet reactivity may predict adverse clinical events, such as ischemia or bleeding, and therefore evidence of increased or decreased platelet reactivity may be noteworthy in some patients. Historically, patients at high risk for thrombotic events included patients with acute coronary syndrome (ACS) undergoing percutaneous coronary intervention (PCI). In a similar fashion, patients undergoing cerebrovascular procedures are also at high risk for thromboembolic or ischemic events. Consequently, DAT is initiated to prevent thromboembolic events, but the risk for thromboembolic events remains in some patients. This increased risk is likely due to differences in stent dynamics when comparing intracranial and coronary stents, as well as high interpatient pharmacological variability with antiplatelet medications, mainly with clopidogrel therapy. The major limitation with clopidogrel therapy is the incomplete activation of its parent compound due to genetic polymorphism, drug-drug interactions, or clinical factors known to increase platelet reactivity. This phenomenon is known as high on-treatment platelet reactivity (HTPR). ${ }^{63}$ Conversely, low on-treatment platelet reactivity (LTPR) may also be observed with clopidogrel or other P2Y12 receptor antagonist therapy due to either a genetic polymorphism or the high potency of antiplatelet effects. HTPR and LTPR with P2Y12 receptor antagonist therapy have been associated with ischemic and bleeding complications, respectively. ${ }^{7,68}$

Incomplete or excess bioactivation of clopidogrel has been implicated in high or low platelet reactivity, respectively, with genetic polymorphism of cytochrome P450 (CYP) isoenzymes 2C19, 3A4, 3A5, 2C9, 1A2, 2B6, paroxonase 1, and ATP-binding cassette efflux transporter. ${ }^{30}$ This led the FDA to revise the prescribing information for clopidogrel in 2010 to include a boxed warning to inform clinicians regarding the potentially detrimental clinical effects of "poor metabolizers" in patients undergoing PCI. There are more than 25 known variant alleles of CYP2C19, but CYP2C19*2 is the most commonly occurring loss-offunction allele..$^{59-61}$ It occurs with high frequency among different ethnic groups, i.e., $12 \%$ in Caucasians, $15 \%$ in African Americans, and 29\%-35\% in Asians. Reduced or absent activity is also observed with alleles $* 3-* 8$, which are found in less than $1 \%$ of all ethnic groups, except for Asians, where $* 3$ is found in $2 \%-9 \%$ of the population. ${ }^{60}$
Other significant mutations to note are functional activity allele $* 1$ and increased activity allele $* 17$. The degree of CYP2C19 function depends on the genotype, whether it is homozygous or heterozygous alleles, as it will determine whether a patient is an ultrarapid metabolizer (e.g., $* 1 / * 17, * 17 / * 17$ ), extensive metabolizer (e.g., *1/*1), intermediate metabolizer (e.g., *1/*2,*1/*3,*2/*17), or poor metabolizer (e.g., $* 2 / 2, * 2 / * 3, * 3 / * 3$ ). Patients who are ultrarapid metabolizers and poor metabolizers are of clinical importance and are identified in 5\%-30\% of all populations and $2 \%-15 \%$ of all populations (specifically $2 \%-5 \%$ of Caucasians and African Americans, and 15\% of Asians), respectively. Observational cohort studies and meta-analyses to date involving patients with ACS undergoing PCI have characterized the increased incidence of major adverse cardiac events, especially stent thrombosis, in patients who were categorized as poor metabolizers ( $* 2$ carrier, $* 1 * 2, * 2 * 2$ ). Conversely, an increased incidence of bleeding was demonstrated in patients who were categorized as ultrarapid metabolizers $\left(* 17\right.$ carrier). ${ }^{30}$ Paré et al. reported conflicting results on clinical outcomes of CYP2C19 genetic variations in patients with ACS without ST-segment elevation (Clopidogrel in Unstable Angina to Prevent Recurrent Events [CURE] trial) and in patients with atrial fibrillation (Atrial Fibrillation Clopidogrel Trial with Irbesartan for Prevention of Vascular Events [ACTIVE] A trial); however, the study is limited by the low incidence of metabolizer phenotypes. ${ }^{49}$

The American Heart Association and the American College of Cardiology Foundation do not recommend routine CYP2C19 genetic testing in all patients due to many clinical concerns. ${ }^{27}$ These concerns include lack of prospective, randomized clinical trials to support the effect of CYP2C19 on clinical outcomes, wide variation in CYP2C19 genetic polymorphisms in the general population, the cost and reimbursement issues associated with genetic testing, interpreting the pharmacogenetics test result, availability of other thienopyridine P2Y12 ADP receptor antagonists, and utilizing the platelet function test as an alternative method of monitoring therapy. However, their clinical expert consensus document suggests genetic testing may benefit patients at high risk for thrombotic complications (e.g., patients with prior stent thrombosis, multivessel PCI procedures, and/or other risk factors associated with high platelet reactivity). With the current body of evidence, the Clinical Pharmacogenetics Implementation Consortium Guideline suggests using standard dosing of clopidogrel if a high-risk patient with ACS is undergoing a PCI procedure and is identified as an ultrarapid or extensive metabolizer, or changing to another thienopyridine P2Y12 ADP receptor antagonist if the patient is identified as an intermediate or poor metabolizer. ${ }^{61}$ Although point-of-care testing for genetic variation is currently unavailable, there are 4 commercially available tests approved by the Center for Devices and Radiological Health (Spartan RX CYP2C19 Test System by Spartan Bioscience, Inc.; Verigene CYP2C19 Nucleic Acid Test by Nanosphere, Inc.; INFINITI CYP2C19 Assay by AutoGenomics, Inc.; and Roche AmpliChip CYP450 microarray by Roche Molecular Systems, Inc.). Similar concepts may be applied to patients undergoing complex neurovascu- 
lar intervention in which CYP2C19 genotyping may be clinically important in a subset of patients. Alternatively, a platelet function test may be used to monitor clopidogrel therapy, although there is no strong evidence to support modifying clopidogrel dose accordingly.

Clinically, variances in platelet reactivity outside acceptable parameters are determined by performing a platelet function test. Based on the predetermined cutoff values, a patient can be categorized as a hypo- or hyperresponder or a nonresponder or as resistant to antiplatelet therapy. Nonresponsiveness or resistance refers to the inability of a medication to inhibit platelet function, so that the medication has a null laboratory and clinical effect. ${ }^{7}$ This can be measured by comparing the results of the platelet function test before and after the drug exposure; however, this may not be a good prognostic indicator due to variable baseline platelet function among patients. Thus, the term "hyporesponsiveness" is used more commonly in clinical practice as the quality indicator of HTPR during antiplatelet therapy. Correspondingly, the term "hyperresponsiveness" is used to identify patients with LTPR during antiplatelet therapy.

\section{Platelet Function Tests}

Platelet reactivity during antiplatelet therapy (also known as residual platelet reactivity) may be quantified with the use of a platelet function test. The gold standard method is light/optical transmission aggregometry (LTA) because it can provide comprehensive platelet function evaluation, where it can be used to diagnose various platelet function disorders and bleeding disorders ${ }^{48}$ When LTA was performed to predict clinical outcomes at 1-year follow-up in patients undergoing elective coronary stent implantation-which included all-cause death, nonfatal acute myocardial infarction, stent thrombosis, and ischemic stroke - the negative and positive predictive values were $94 \%$ and $11.7 \%$, respectively, with sensitivity and specificity of $60.2 \%$ and $59.1 \%$, respectively (using $5 \mu \mathrm{mol} / \mathrm{L}$ ADP as the agonist). ${ }^{8}$ However, its clinical use has been limited mainly due to lack of standardization and the timeconsuming preparation process. The main issues include choosing an agonist or activator and its specific concentration, as well as the preparation of either platelet-rich or platelet-poor plasma. Ideally, point-of-care testing should be rapid, require low sample volume, minimize sample manipulation or preparation, and correlate well with LTA. Newer methods for point-of-care testing are now available, with advantages that include semiautomation, low required sample volume, and minimal to no required sample preparation..$^{48,54,70}$ In addition to many technical variations in each of the platelet function tests, there also appears to be variation in clinical utility as some are used to monitor antiplatelet treatment effects, screen primary hemostasis, diagnose platelet dysfunction, predict bleeding risk, and guide transfusion therapy. ${ }^{41}$ Semiautomated tests include multiple electrode aggregometry, Multiplate (Roche Diagnostics), Platelet Works (Helena Laboratories), vasodilator-stimulated phosphoprotein phosphorylation, Cone and Plate(let) assay, and IMPACT-R (Daned SA). The clinical utility of these tests is limited by additional sample preparation, the requirement of an experienced technician, and the limited clinical data available in monitoring antiplatelet therapy. Automated tests include Platelet Function Analyzer (PFA-100; Siemens Healthcare Diagnostics) and VerifyNow (Accriva Diagnostics). PFA-100 may be useful in detecting platelet dysfunction in patients without antiplatelet therapy, as in von Willebrand disease, and assessing the effects of aspirin therapy. However, this test does not correlate well with thienopyridine therapy and may be of limited value in some clinical settings. In comparison, VerifyNow may not be useful in detecting primary hemostasis but may be useful in assessing antiplatelet treatment effects, including thienopyridine therapy.

The most commonly used point-of-care platelet function testing is VerifyNow, and it correlates well with LTA $(\mathrm{r}=0.67-0.77) .{ }^{21}$ VerifyNow, previously known as Ultegra Rapid Platelet Function Assay, uses a similar concept as LTA to quantify the degree of platelet aggregation as measured by light transmittance. A whole blood sample is required using a 2-ml Greiner partial fill Vacuette tube, and with its placement in the VerifyNow System (an analyzer instrument) containing a disposable, reagent-specific assay device, the result is available within 5-10 minutes (http://www.accriva.com/products/verifynow-systemplatelet-reactivity-test). Platelets in the blood sample are activated with a reagent of choice and aggregate around fibrinogen-coated beads. The degree of platelet aggregation in this final chamber changes light transmittance, where a decrease in light transmittance (expressed as low unit of measurement) indicates increased platelet function inhibition (or good response to antiplatelet therapy). Different reagent-specific assay devices can be used to assess the effects of aspirin, P2Y12 inhibitor, or glycoprotein IIb/ IIIa inhibitor on platelet function and are expressed as aspirin reaction units (ARUs), $\mathrm{P} 2 \mathrm{Y} 12$ reaction units (PRUs), or platelet aggregation units, respectively.

The association between clinical outcomes and residual platelet reactivity in patients undergoing neuroendovascular procedures requiring DAT has been explored in small, retrospective, single-center studies (Table 1). VerifyNow was most commonly used as the platelet function test due to the ease of its use and validation with the LTA. Most of these studies were published in the last 5 years. The collective interpretation of these study results is challenging due to many confounding variables. The variables include the neurointerventionalists' surgical experience, nonstandardized antiplatelet regimens, variable definitions for HTPR and LTPR, and different definitions for adverse clinical events.

There are 4 studies utilizing receiver operating characteristic analyses to identify optimal cutoffs for best predicting thromboembolic and bleeding events based on platelet function analysis (Table 1). ${ }^{22,33,34,44}$ Each of these studies used VerifyNow with the cutoff values established using either PRUs or P2Y12 percentage inhibition. Kang et al. and Nishi et al. found cutoff values for ischemic and bleeding events, respectively, but the specificity and sensitivity were low. ${ }^{33,44}$ Goh et al. and Kashiwazaki et al. found similar cutoff values for major bleeding events with high sensitivity and specificity, 22,34 but the clinical application of P2Y12 percentage inhibition in assessing antiplatelet therapy remains controversial. This is primarily due to the 
TABLE 1. Receiver operating characteristic analysis in endovascular neurosurgery

\begin{tabular}{llllrc}
\hline \multicolumn{1}{c}{ Authors \& Year } & \multicolumn{1}{c}{ Cutoff Value } & \multicolumn{1}{c}{ End Point } & AUC (95\% Cl) & \% Sensitivity & \% Specificity \\
\hline Kang et al., 2010 & PRU >295 & TE & $0.675(0.526-0.825)$ & 75 & 57 \\
\hline Goh et al., 2013 & $\geq 72 \%$ inhibition & Major bleed & $0.96(0.89-1)$ & 100 & 90.9 \\
& $\geq 53 \%$ inhibition & Major/minor bleed & $0.75(0.58-0.93)$ & 70 & 75.7 \\
\hline Kashiwazaki et al., 2014 & $\leq 26 \%$ inhibition & TE; major bleed & $0.79(0.65-0.93)$ & 81.1 & 69.2 \\
& $\geq 74 \%$ inhibition & & $0.82(0.71-0.98)$ & 86.2 & 87.5 \\
\hline Nishi et al., 2016 & PRU $\leq 175$ & Bleed & 0.63 & 54 & 72 \\
\hline
\end{tabular}

$\mathrm{AUC}=$ area under the curve; $\mathrm{TE}=$ thromboembolism.

VerifyNow was the platelet function test used in each of the 4 studies.

lack of association between P2Y12 percentage inhibition and clinical outcomes, ${ }^{33}$ the inability to make the P2Y12 percentage inhibition correspond with PRU, and the dependence of P2Y12 percentage inhibition on a patient's baseline platelet function value. These varied results led Accriva Diagnostics in August 2012 to voluntarily withdraw in North America the reporting of P2Y12 percentage inhibition to accurately assess and reflect platelet function during antiplatelet therapy.

\section{Summary of Experience With Antiplatelet Agents Used in Neuroendovascular Procedures}

Antiplatelet agents are necessary for patients undergoing neuroendovascular procedures, particularly intracranial stenting and aneurysm coiling. Evaluation of the classic dual antiplatelet combination of aspirin and clopidogrel constitutes the majority of the published literature in this area (Table 2). ${ }^{9}$ Currently, the combination of aspirin and clopidogrel is recommended prior to neuroendovascular stent deployment, although there is considerable heterogeneity in treatment. ${ }^{19}$ The duration of therapy tends to be longer than that for bare metal stent deployment in PCI, with most practitioners prescribing DAT for at least 3 months. Numerous other agents have been investigated to a limited degree in this clinical context, including prasugrel, ticagrelor, cilostazol, and glycoprotein IIb/IIIa inhibitors, such as abciximab and eptifibatide. These agents are primarily used in the case of clopidogrel resistance or, in the case of glycoprotein IIb/IIIa inhibitors, acute procedural thrombosis. The use of more than 1 antiplatelet agent in neuroendovascular procedures permits the prescriber to take advantage of the additive effects of these agents. Several mechanisms of action come into play when combining antiplatelet agents, including inhibition of thromboxane, the P2Y12 ADP receptor, cyclic AMP, and the glycoprotein IIb/IIIa receptor (Table 3).

\section{Aspirin}

Aspirin is the most common component of DAT in patients undergoing neuroendovascular procedures. Aspirin is widely available, well tolerated, and has extensive clinical evidence supporting its use in the cardiovascular arena. Aspirin is typically initiated at least 3-5 days prior to carotid or intracranial stenting, although many practitioners may start therapy as long as 14-21 days prior to a procedure. In emergency neuroendovascular interventions in which preprocedure aspirin therapy is not possible, loading doses have varied from 200 to $650 \mathrm{mg}$. Based on various laboratory definitions for HTPR, the incidence of aspirin hyporesponsiveness varied from $2.1 \%$ to $13.5 \%{ }^{5,17,20,36 \text {, }}$ 47,53 Prabhakaran et al. found a strong, inverse relationship between preprocedural weekly aspirin dose and ARUs, in which lower aspirin doses were associated with higher ARUs.$^{53}$ Their findings suggest that aspirin should be given at a dose of at least $100 \mathrm{mg}$ daily to maintain ARUs $\leq 550$. While the antiplatelet effect of aspirin in some individuals may be attenuated, there is no evidence to suggest that this affects outcomes. Likewise, no evidence exists suggesting higher doses of aspirin (> $325 \mathrm{mg} /$ day) or alternative antiplatelet agents are necessary in these cases. Nearly all studies investigating antiplatelet therapy in patients undergoing neuroendovascular procedures include aspirin as part of the antiplatelet therapy regimen, with doses ranging from 81 to $325 \mathrm{mg}$ daily. $2,16,17,20,22,25,28,34-36,43-47,66,70$

\section{P2Y12 Inhibitors \\ Clopidogrel}

Clopidogrel is the other most common component of DAT in patients undergoing neuroendovascular procedures. Variation in individual response to clopidogrel has sparked a great deal of research and controversy in the cardiovascular and neuroendovascular areas. The incidence of clopidogrel hyporesponsiveness has varied from $21 \%$ to $53.1 \%$ in patients undergoing neuroendovascular procedures. ${ }^{5,15-17,20,34-36,43,45,47,53,70}$ The use of clopidogrel after coronary interventions is well described, although currently the role of clopidogrel resistance and platelet function testing after PCI is not definitive. The Gauging Responsiveness with a VerifyNow P2Y12 assay: Impact on Thrombosis and Safety (GRAVITAS) study, which evaluated clopidogrel response in 2214 patients with HTPR undergoing PCI with drug-eluting stents, suggested that high-dose clopidogrel was no more effective than standard dose clopidogrel. However, subgroup analyses indicated that achievement of a target PRU $(<208)$ was associated with a lower risk for cardiovascular events..$^{55,56}$ Asymptomatic and symptomatic thromboembolic events after neuroendovascular intervention have been primarily associated with clopidogrel hyporesponsiveness; this issue can result in events as high as $7.1 \%$ within 24 hours of the procedure, $41 \%$ within 30 days of the procedure, and 


\section{K. S. Kim et al.}

TABLE 2. Outcomes in clopidogrel hyporesponders

\begin{tabular}{|c|c|c|c|c|c|c|}
\hline \multirow{2}{*}{$\begin{array}{l}\text { Authors } \\
\& \text { Year }\end{array}$} & \multirow{2}{*}{$\begin{array}{l}\text { No. of Patients } \\
\text { (study dates)* }^{*}\end{array}$} & \multicolumn{2}{|c|}{ Treatment } & \multirow{2}{*}{$\begin{array}{l}\text { Method \& Definition } \\
\text { of Hyporesponder }\end{array}$} & \multicolumn{2}{|r|}{ Outcome } \\
\hline & & Neuroendovascular & Antiplatelet & & Laboratory & Clinical \\
\hline $\begin{array}{l}\text { Lee et al., } \\
2008\end{array}$ & $\begin{array}{c}98(6 / 2006- \\
4 / 2007)\end{array}$ & $\begin{array}{l}\text { Stent (intracranial } \\
\text { aneurysms, in- } \\
\text { tra- \& extra- } \\
\text { cranial artery } \\
\text { stenosis) }\end{array}$ & $\begin{array}{l}\text { CLP } 300 \text { mg LD (600 } \\
\text { mg LD if same day or } \\
300 \text { mg LD if P2Y12 } \\
\% \text { inhibition }<40 \%) / 75 \\
\text { mg daily + ASA } 325 \\
\text { mg daily, } 5-10 \text { days } \\
\text { prior }\end{array}$ & $\begin{array}{l}\text { VerifyNow prior to } \\
\text { procedure; ARU } \\
\quad<550, \mathrm{P} 2 Y 12 \% \\
\text { inhibition } \geq 40 \%\end{array}$ & $\begin{array}{l}\text { Aspirin poor } \\
\text { respond- } \\
\text { ers } 2.1 \% \\
(2 / 95), \\
\text { CLP poor } \\
\text { respond- } \\
\text { ers } 42.9 \% \\
(42 / 98)\end{array}$ & $\begin{array}{l}\text { Clinical outcome defined in this } \\
\text { study as intraprocedural throm- } \\
\text { bosis \& thromboembolic events: } \\
\text { overall asymptomatic TE events } \\
\text { 3.1\% (3/98); CLP poor respond- } \\
\text { ers, asymptomatic TE events } \\
\text { 7.1\% (3/42); no bleeding event } \\
\text { data available }\end{array}$ \\
\hline $\begin{array}{l}\text { Müller- } \\
\text { Schunk } \\
\text { et al., } \\
2008\end{array}$ & 50 & $\begin{array}{l}\text { Stent (extracranial } \\
\text { stenosis, intra- } \\
\text { cranial stenosis) }\end{array}$ & $\begin{array}{l}\text { ASA } 100 \text { mg + CLP } 300 \\
\text { mg LD } \geq 12 \text { hrs prior, } \\
75 \text { mg daily (or >48 } \\
\text { hrs prior); intraproce- } \\
\text { dure: heparin IV (ACT } \\
\text { 200-300 sec); IV tirofi- } \\
\text { ban to dissolve clot }\end{array}$ & $\begin{array}{l}\text { Multiplate analyzer } \\
\text { prior to proce- } \\
\text { dure; } \leq 52 \text { ARU }\end{array}$ & $\begin{array}{l}\text { ASA nonre- } \\
\text { sponders } \\
0, \text { CLP } \\
\text { nonre- } \\
\text { sponders } \\
28 \% \\
(14 / 50)\end{array}$ & $\begin{array}{l}\text { Overall transient intrainterventional } \\
\text { thrombosis 2; overall TIA or } \\
\text { infarction 3; adverse events in } \\
\text { nonresponders vs responders } \\
42 \%(5 / 12) \text { vs } 0 \%(0 / 36), p= \\
0.001\end{array}$ \\
\hline $\begin{array}{l}\text { Prabha- } \\
\text { karan } \\
\text { et al., } \\
2008\end{array}$ & $\begin{array}{c}76(5 / 2005- \\
8 / 2006)\end{array}$ & $\begin{array}{l}\text { Stents (intracra- } \\
\text { nial aneurysms, } \\
\text { intra- \& extra- } \\
\text { cranial artery } \\
\text { stenosis) }\end{array}$ & $\begin{array}{l}\text { ASA alone 16/76 } \\
\text { (21.0\%), CLP alone } \\
\text { 4/76 (5.3\%), or ASA } \\
\text { + CLP 56/76 (73.7\%), } \\
\text { loaded w/in } 1 \mathrm{wk} \\
\text { prior; intraprocedure: } \\
\text { IV heparin (ACT } \\
\text { 250-300 sec) }\end{array}$ & $\begin{array}{l}\text { VerifyNow prior to } \\
\text { procedure; ARU } \\
<550, \mathrm{P} 2 \mathrm{Y} 12 \% \\
\text { inhibition }>40 \%\end{array}$ & $\begin{array}{l}\text { ASA low } \\
\text { respond- } \\
\text { ers } 4.2 \% \\
(3 / 71) \\
\text { CLP low } \\
\text { respond- } \\
\text { ers } 50.9 \% \\
(28 / 55)\end{array}$ & $\begin{array}{l}\text { Intraprocedure acute stent throm- } \\
\text { bosis or stenosis 0; intraproce- } \\
\text { dure aneurysmal rupture 1; w/ } \\
\text { in } 6 \text { mos, TIA } 3.9 \% \text { (3/76); no } \\
\text { bleeding event data available }\end{array}$ \\
\hline $\begin{array}{l}\text { Kang et } \\
\text { al., } \\
2010\end{array}$ & $\begin{array}{l}\text { 186: } 209 \\
\text { aneurysms } \\
(10 / 2008)\end{array}$ & $\begin{array}{l}\text { Coil embolization } \\
\text { (ICA, ACA, } \\
\text { MCA, PCA) } \pm \\
\text { stent }\end{array}$ & $\begin{array}{l}\text { Intraprocedure: IV hepa- } \\
\text { rin } 3000 \text { units bolus, } \\
\text { then } 1000 \text { units/hr }\end{array}$ & $\begin{array}{l}\text { VerifyNow prior to } \\
\text { procedure }\end{array}$ & & $\begin{array}{l}\text { Overall procedure-related TE } \\
\text { events } 9.1 \%(17 / 186) ; \text { intra- } \\
\text { procedure TE events } 4.8 \% \\
\text { (9/186); postprocedure ischemic } \\
\text { events } 2.7 \% \text { (5/186); procedural } \\
\text { aneurysmal perforations } 1.6 \% \\
\text { (3/186); procedure-related per- } \\
\text { manent morbidity or mortality } 0\end{array}$ \\
\hline $\begin{array}{l}\text { Pandya } \\
\text { et al., } \\
2010\end{array}$ & $\begin{array}{l}\text { 238: data anal- } \\
\text { ysis based } \\
\text { on } 216 \\
(2 / 2006- \\
11 / 2007)\end{array}$ & $\begin{array}{l}\text { Embolization, } \\
\text { stents (intracra- } \\
\text { nial aneurysm, } \\
\text { carotid stenosis, } \\
\text { other) }\end{array}$ & $\begin{array}{l}\text { ASA } 81 \text { mg daily, CLP } \\
75 \text { mg daily, } \geq 7 \text { days } \\
\text { prior; CLP } 300-600 \\
\text { mg, ASA } 325 \text { mg LD if } \\
\text { emergency }\end{array}$ & $\begin{array}{l}\text { VerifyNow prior to } \\
\text { procedure; ARU } \\
<550, \text { P2Y12 } \% \\
\text { inhibition }>50 \%\end{array}$ & $\begin{array}{l}12 \%(26 / 216) \\
\text { ASA in- } \\
\text { adequate } \\
\text { respond- } \\
\text { er, } 34 \% \\
(74 / 216) \\
\text { CLP in- } \\
\text { adequate } \\
\text { responder }\end{array}$ & \\
\hline $\begin{array}{l}\text { Drazin } \\
\text { et al., } \\
2011\end{array}$ & $\begin{array}{l}52(2007- \\
2009)\end{array}$ & $\begin{array}{l}\text { Stent (intracra- } \\
\text { nial stenosis, } \\
\text { cervical carotid } \\
\text { artery stenosis, } \\
\text { intracranial } \\
\text { aneurysms) }\end{array}$ & $\begin{array}{l}\text { CLP } 600 \text { mg LD (subop- } \\
\text { timal responders } 300 \\
\text { mg LD if } 10 \%-19 \% \text {, } \\
600 \mathrm{mg} \text { LD if }<10 \% \text { ) } \\
75 \mathrm{mg} \text { daily, } \geq 12 \mathrm{hrs} \\
\text { prior; ASA } 81 \mathrm{mg} \text { daily } \\
\text { day prior }\end{array}$ & $\begin{array}{l}\text { VerifyNow prior to } \\
\text { procedure; ARU } \\
\leq 550, \text { P2Y12 } \% \\
\text { inhibition } \geq 20 \%\end{array}$ & $\begin{array}{l}\text { ASA sub- } \\
\text { optimal } \\
\text { respond- } \\
\text { ers } 7 / 52 \\
(13.5 \%) \\
\text { CLP sub- } \\
\text { optimal } \\
\text { respond- } \\
\text { ers } 19 / 52 \\
(36.5 \%)\end{array}$ & $\begin{array}{l}\text { TE events in CLP suboptimal } \\
\text { responders vs optimal respond- } \\
\text { ers } 1 \text { vs } 0\end{array}$ \\
\hline $\begin{array}{l}\text { Koerner } \\
\text { et al., } \\
2012\end{array}$ & $\begin{array}{c}44(8 / 2008- \\
6 / 2009)\end{array}$ & $\begin{array}{l}\text { Stent (extracranial, } \\
\text { intracranial), } \\
\text { percutaneous } \\
\text { transluminal } \\
\text { angioplasty }\end{array}$ & $\begin{array}{l}\text { ASA } 100 \text { mg daily, CLP } \\
75 \text { mg daily, } \geq 3 \text { days } \\
\text { prior; ASA } 500 \text { mg } \\
\text { LD, CLP } 300 \text { mg LD } \\
\text { if urgent }\end{array}$ & $\begin{array}{l}\text { Multiplate analyzer } \\
\text { prior to proce- } \\
\text { dure; } \leq 468 \text { ARU } \\
\text { min }\end{array}$ & $\begin{array}{l}\text { Hypore- } \\
\text { sponders } \\
25 \% \\
(11 / 44)\end{array}$ & $\begin{array}{l}\text { Overall new DWI lesions } 36 \% \\
\text { (16/44), new DWI lesions in } \\
\text { hyporesponders vs responders } \\
36 \%(4 / 11) \text { vs } 33 \%(11 / 33)\end{array}$ \\
\hline
\end{tabular}


» CONTINUED FROM PAGE 894

TABLE 2. Outcomes in clopidogrel hyporesponders

\begin{tabular}{|c|c|c|c|c|c|c|}
\hline \multirow{2}{*}{$\begin{array}{l}\text { Authors } \\
\text { \& Year }\end{array}$} & \multirow{2}{*}{$\begin{array}{l}\text { No. of Patients } \\
\text { (study dates) }^{*}\end{array}$} & \multicolumn{2}{|c|}{ Treatment } & \multirow{2}{*}{$\begin{array}{l}\text { Method \& Definition } \\
\text { of Hyporesponder }\end{array}$} & \multicolumn{2}{|r|}{ Outcome } \\
\hline & & Neuroendovascular & Antiplatelet & & Laboratory & Clinical \\
\hline $\begin{array}{l}\text { Delgado } \\
\text { Alman- } \\
\text { doz } \\
\text { et al., } \\
2013\end{array}$ & $\begin{array}{l}\text { 44: } 48 \text { PED } \\
\text { proce- } \\
\text { dures, } 54 \\
\text { aneurysms } \\
(11 / 17 / 2011- \\
7 / 23 / 2012)\end{array}$ & $\begin{array}{l}\text { PED (intracranial } \\
\text { aneurysm) }\end{array}$ & $\begin{array}{l}\text { ASA } 325 \text { mg daily + CLP } \\
75 \text { mg daily (prasugrel } \\
60 \text { mg LD, then } 10 \mathrm{mg} \\
\text { daily if CLP hypore- } \\
\text { sponder; CLP dose } \\
\text { reduced if hyperre- } \\
\text { sponder; if prasugrel } \\
\text { hyporesponder, then } \\
\text { received ticagrelor } \\
180 \text { mg, then } 90 \text { mg } \\
2 x / \text { day; if prasugrel } \\
\text { hyperresponder, } \\
\text { then prasugrel dose } \\
\text { reduced; prasugrel } \\
60 \text { mg LD, then } 10 \\
\text { mg daily, if urgent/ } \\
\text { emergency) } 10 \text { days } \\
\text { prior; intraprocedure: } \\
\text { heparin (ACT 2-2.5 } \\
\text { times baseline) }\end{array}$ & $\begin{array}{l}\text { VerifyNow prior to } \\
\text { procedure; PRU } \\
80-200\end{array}$ & $\begin{array}{l}\text { CLP hypore- } \\
\text { sponders } \\
\text { (PRU } \\
>200) \\
26.2 \% \\
(11 / 42) ; \\
\text { CLP } \\
\text { hypore- } \\
\text { sponders } \\
\text { (PRU } \\
>240 \text { ) } \\
21 \% ; \text { CLP } \\
\text { hyperre- } \\
\text { sponders } \\
\text { (PRU } \\
<80 \text { ) } \\
21.4 \% \\
\text { (9/42); } \\
\text { CLP } \\
\text { hyperre- } \\
\text { sponders } \\
\text { (PRU } \\
<60) 14 \%\end{array}$ & $\begin{array}{l}\text { Normal response = PRU 60-240; } \\
\text { TE events } 8.3 \% \text { (4/48), bleeding } \\
\text { events } 8.3 \%(4 / 48) \text {, major hem- } \\
\text { orrhagic complications in ASA/ } \\
\text { prasugrel } 16.7 \% \text { vs ASA/CLP } \\
2.9 \%, p=0.16\end{array}$ \\
\hline $\begin{array}{c}\text { Goh et al., } \\
2013\end{array}$ & $\begin{array}{c}47(5 / 2010- \\
5 / 2011)\end{array}$ & $\begin{array}{l}\text { Coil emboliza- } \\
\text { tion, balloon } \\
\text { remodeling, } \\
\text { stent assistance, } \\
\text { stent (intracra- } \\
\text { nial aneurysm, } \\
\text { stenosis) }\end{array}$ & $\begin{array}{l}\text { ASA } 100 \text { mg daily, CLP } \\
75 \text { mg daily, } 3 \text { days } \\
\text { prior; intraprocedure: } \\
\text { IV heparin (ACT } 2 \\
\text { times normal); post- } \\
\text { procedure: IV heparin } \\
\text { (aPTT titration) }\end{array}$ & $\begin{array}{l}\text { VerifyNow prior to } \\
\text { procedure; major } \\
\text { bleed } \geq 72 \% \\
\text { inhibition, major } \\
\text { \& minor bleed } \\
\geq 53 \% \text { inhibition }\end{array}$ & $\begin{array}{l}\text { Hyperre- } \\
\text { sponders } \\
(\geq 72 \% \\
\text { inhibition) } \\
14.9 \% \\
(7 / 47)\end{array}$ & $\begin{array}{l}\text { Overall bleeding event during } \\
\text { procedure } 23.4 \% \text { (11/47); w/in } 24 \\
\text { hrs postprocedure } 6.4 \% \text { (3/47); } \\
\text { TIMI major bleeding events } ~ \\
14.9 \text { (7/47); TIMI minor bleeding } \\
\text { events, small groin hematomas } \\
\text { of little clinical significance; } \\
\text { major bleeding events in hyper- } \\
\text { responders } 6.4 \% \text { (3/47) }\end{array}$ \\
\hline $\begin{array}{c}\text { Fifi et al., } \\
2013\end{array}$ & $\begin{array}{l}\text { Group A: } 49 \\
\text { (9/2006- } \\
\text { 9/2008); } \\
\text { Group B: } 47 \\
\text { (10/2008- } \\
\text { 1/2011; } \\
\text { additional } \\
\text { 150-600 mg } \\
\text { CLP prior to } \\
\text { procedure if } \\
\text { resistant) }\end{array}$ & $\begin{array}{l}\text { Stent (extra-/intra- } \\
\text { cranial stenosis, } \\
\text { intracranial } \\
\text { aneurysm) }\end{array}$ & $\begin{array}{l}\text { ASA } 81 \text { mg daily + CLP } \\
75 \text { mg daily ( } 600 \mathrm{mg} \\
\text { LD if emergency), } 5 \\
\text { days prior; intraproce- } \\
\text { dure: IV heparin (ACT } \\
2 \text { times baseline) }\end{array}$ & $\begin{array}{l}\text { VerifyNow prior to } \\
\text { procedure; ARU } \\
<550, \text { P2Y12 \% } \\
\text { inhibition }>20 \%\end{array}$ & $\begin{array}{l}\text { ASA resis- } \\
\text { tant } 5.3 \% \\
(5 / 95) \\
\text { CLP resis- } \\
\text { tant } 37.5 \% \\
(36 / 96)\end{array}$ & $\begin{array}{l}\text { Overall TE events } 7.3 \%(7 / 96) \\
\text { TE events in CLP resistant vs } \\
\text { nonresistant } 16.7 \%(6 / 36) \text { vs } \\
1.6 \%(1 / 60), p<0.01\end{array}$ \\
\hline $\begin{array}{l}\text { Heller } \\
\text { et al., } \\
2013\end{array}$ & $\begin{array}{l}\text { 24: } 27 \text { aneu- } \\
\text { rysms, } 25 \\
\text { procedures } \\
\text { (10/2011- } \\
6 / 2012)\end{array}$ & PED (ICA) & $\begin{array}{l}\text { ASA \& CLP } 75 \text { mg daily, } \\
\geq 7 \text { days prior; intra- } \\
\text { procedure: IV heparin } \\
\text { (aPTT } 240 \text { sec); } \\
\text { postprocedure: IV } \\
\text { heparin (aPTT 50-70 } \\
\text { sec) for } 12-18 \text { hrs }\end{array}$ & $\begin{array}{l}\text { Light transmission } \\
\text { aggregometry } \\
\text { prior to proce- } \\
\text { dure; ASA max } \\
\text { platelet aggrega- } \\
\text { tion } \leq 20 \% \text {, CLP } \\
\text { MPA } \leq 60 \%\end{array}$ & $\begin{array}{l}\text { ASA nonre- } \\
\text { sponders } \\
16 \% \\
(4 / 25) \\
\text { CLP } \\
\text { nonre- } \\
\text { sponders } \\
4 \%(1 / 25)\end{array}$ & $\begin{array}{l}\text { By Day } 1 \text { asymptomatic (MR-DWI) } \\
\text { ischemia in all patients } 52 \% \\
(13 / 25)\end{array}$ \\
\hline
\end{tabular}




\section{K. S. Kim et al.}

\section{» CONTINUED FROM PAGE 895}

TABLE 2. Outcomes in clopidogrel hyporesponders

\begin{tabular}{|c|c|c|c|c|c|c|}
\hline \multirow{2}{*}{$\begin{array}{l}\text { Authors } \\
\& \text { Year }\end{array}$} & \multirow{2}{*}{$\begin{array}{l}\text { No. of Patients } \\
\text { (study dates) }\end{array}$} & \multicolumn{2}{|c|}{ Treatment } & \multirow{2}{*}{$\begin{array}{l}\text { Method \& Definition } \\
\text { of Hyporesponder }\end{array}$} & \multicolumn{2}{|r|}{ Outcome } \\
\hline & & Neuroendovascular & Antiplatelet & & Laboratory & Clinical \\
\hline $\begin{array}{l}\text { Nordeen } \\
\text { et al., } \\
2013\end{array}$ & $\begin{array}{c}81(10 / 2009- \\
9 / 2010)\end{array}$ & $\begin{array}{l}\text { Angioplasty \& } \\
\text { stent, stent-as- } \\
\text { sisted coil, stent, } \\
\text { other (procedure } \\
\text { performed only } \\
\text { at P2Y12 \% inhi- } \\
\text { bition } \geq 20 \% \text { ) }\end{array}$ & $\begin{array}{l}\text { ASA } 325 \text { mg daily (650 } \\
\text { mg LD if emer- } \\
\text { gency) + CLP } 75 \text { mg } \\
\text { daily ( } 300-600 \text { mg } \\
\text { LD if emergency or } \\
\text { resistant or change to } \\
\text { ticlopidine, prasugrel, } \\
\text { or ticagrelor), 5-7 } \\
\text { days prior, then for } 1 \\
\text { mo after procedure; } \\
\text { intraprocedure: hepa- } \\
\text { rin or glycoprotein IIb/ } \\
\text { Illa antagonist IV }\end{array}$ & $\begin{array}{l}\text { VerifyNow prior } \\
\text { to procedure; } \\
\text { P2Y12 \% } \\
\text { inhibition } \geq 20 \% \\
\text { (CLP resistant if } \\
\text { P2Y12 \% inhibi- } \\
\text { tion }<20 \% \text { after } 2 \\
\text { tests) }\end{array}$ & $\begin{array}{l}\text { CLP resis- } \\
\text { tant } 21 \% \\
(17 / 81)\end{array}$ & $\begin{array}{l}\text { W/in } 24 \text { hrs of procedure: ischemia } \\
0 \% \text { vs } 1 \% \text {, hemorrhage } 6 \% \\
\text { vs } 8 \%, p=N S \text {; by } 30 \text { days, } \\
\text { ischemia } 0 \text { vs } 3 \% \text {, hemorrhage } \\
9 \% \text { vs } 8 \%, p=N S \text {; by } 90 \text { days, } \\
\text { ischemia } 0 \text { vs } 8 \% \text {, hemorrhage } \\
9 \% \text { vs } 8 \%, p=\text { NS }\end{array}$ \\
\hline $\begin{array}{l}\text { Delgado } \\
\text { Alman- } \\
\text { doz } \\
\text { et al., } \\
2014\end{array}$ & $\begin{array}{l}\text { 44: } 48 \text { PED } \\
\text { proce- } \\
\text { dures, } 54 \\
\text { aneurysms } \\
(11 / 17 / 2011- \\
7 / 23 / 2012)\end{array}$ & $\begin{array}{l}\text { PED (intracranial } \\
\text { aneurysm) }\end{array}$ & Same as above & $\begin{array}{l}\text { VerifyNow prior } \\
\text { to procedure; } \\
10 \& 30 \text { days } \\
\text { after change in } \\
\text { P2Y12 receptor } \\
\text { antagonist thera- } \\
\text { py, after change } \\
\text { to medications } \\
\text { that may affect } \\
\text { CLP metabolism, } \\
\text { or any time if TE/ } \\
\text { hemorrhage; } \\
\text { PRU } 80-200\end{array}$ & $\begin{array}{l}\text { CLP } \\
\text { hyperre- } \\
\text { sponders } \\
\text { (PRU } \\
<80 \text { ) } \\
62 \% \text {, CLP } \\
\text { hyperre- } \\
\text { sponders } \\
\text { (PRU } \\
<60) 50 \%\end{array}$ & $\begin{array}{l}\text { Normal response = PRU 60-240; } \\
\text { overall TE events 12.5\% (6/48); } \\
\text { TE events in CLP hyporesponse } \\
\text { vs nonhyporesponse } 100 \% \\
\text { (2/2) vs } 8.7 \% \text { (4/46); bleeding } \\
\text { events in CLP hyperresponse vs } \\
\text { nonhyperresponse } 44.4 \%(4 / 9) \\
\text { vs } 2.6 \% \text { (1/39); clinical outcome } \\
\text { w/ PRU } 60-240 \text { up to } 6 \text { mos } \\
\text { after Tx: overall } 12.5 \% \text { (6/48) TE, } \\
10.4 \% \text { (5/48) hemorrhagic, major } \\
\text { bleed } 6.2 \% \text { (3/48), parenchymal } \\
\text { ICH/death 4.2\% (2/48); if PRU } \\
<60,60 \% \text { conversion to hyper- } \\
\text { responder }\end{array}$ \\
\hline $\begin{array}{l}\text { Kashiwa- } \\
\text { zaki } \\
\text { et al., } \\
2014\end{array}$ & $\begin{array}{l}\text { 66: } 66 \\
\text { interventions } \\
\text { (10/2011- } \\
\text { 10/2013) }\end{array}$ & $\begin{array}{l}\text { Stent, coil } \pm \text { stent } \\
\quad \text { (carotid steno- } \\
\text { sis, intracranial } \\
\text { aneurysm) }\end{array}$ & $\begin{array}{l}\text { ASA } 100 \text { mg + CLP } 75 \\
\text { mg daily, } \geq 14 \text { days } \\
\text { prior; intraprocedure: } \\
\text { IV heparin (ACT } \\
2 \text { times baseline); } \\
\text { antiplatelet therapy } \\
\text { for } \geq 30 \text { days after } \\
\text { procedure }\end{array}$ & $\begin{array}{l}\text { VerifyNow w/in } 48 \\
\text { hrs of procedure; } \\
26 \%-74 \% \text { inhibi- } \\
\text { tion }\end{array}$ & $\begin{array}{l}\text { Hypore- } \\
\text { sponders } \\
28.8 \% \\
(19 / 66) \\
\text { hyperre- } \\
\text { sponders } \\
22.7 \% \\
(15 / 66)\end{array}$ & $\begin{array}{l}\text { Overall bleeding events } 12.1 \% \\
\text { (8/66); overall asymptom- } \\
\text { atic ischemic events } 19.7 \% \\
\text { (13/66); bleeding event in } \\
\text { hyperresponders vs nonhy- } \\
\text { perresponders: } 6(44.4 \%) \text { in } \\
\text { hyperresponse group, } 2(6.9 \%) \\
\text { in appropriate-response group, } \\
p=0.001 \text {; ischemic events in } \\
\text { hyporesponders vs nonhypore- } \\
\text { sponders } 9 \text { vs } 4, p=0.001\end{array}$ \\
\hline $\begin{array}{l}\text { Oran et } \\
\text { al., } \\
2015\end{array}$ & $\begin{array}{l}\text { 100: } 104 \\
\text { aneurysms, } \\
\text { flow-divert- } \\
\text { ing stents } \\
33 \text { standard } \\
\text { group (no } \\
\text { test) vs } 71 \\
\text { aggregome- } \\
\text { try group } \\
\text { (test); } \\
\text { (1/2010- } \\
\text { 6/2013) }\end{array}$ & $\begin{array}{l}\text { Flow-diverting } \\
\text { stents (intracra- } \\
\text { nial aneurysm) }\end{array}$ & $\begin{array}{l}\text { ASA } 300 \text { mg LD + } \\
\text { CLP } 600 \text { mg LD (if } \\
\text { resistant, ticlopidine } \\
1 \mathrm{~g} \& / \text { or IV tirofiban } \\
\text { or IV heparin higher } \\
\text { dose), 8-12 hrs prior; } \\
\text { intraprocedure: IV } \\
\text { heparin 5000-10,000 } \\
\text { units bolus (ACT 2-3 } \\
\text { times baseline) }\end{array}$ & $\begin{array}{l}\text { Multiplate analyzer } \\
\text { prior to proce- } \\
\text { dure; ASA AUC } \\
\leq 500, \text { CLP AUC } \\
\leq 468\end{array}$ & $\begin{array}{l}\text { CLP hypore- } \\
\text { sponders } \\
25 \% \\
(17 / 68)\end{array}$ & $\begin{array}{l}\text { Symptomatic thrombotic events in } \\
\text { no-test group } 9.1 \%(3 / 33) \text {, death } \\
\text { in no-test group } 3.1 \%(1 / 32) \text {, ma- } \\
\text { jor bleeding event in aggregome- } \\
\text { try group } 2.8 \%(2 / 71)\end{array}$ \\
\hline
\end{tabular}


» CONTINUED FROM PAGE 896

TABLE 2. Outcomes in clopidogrel hyporesponders

\begin{tabular}{|c|c|c|c|c|c|c|}
\hline \multirow{2}{*}{$\begin{array}{l}\text { Authors } \\
\text { \& Year }\end{array}$} & \multirow{2}{*}{$\begin{array}{l}\text { No. of Patients } \\
\text { (study dates) }^{*}\end{array}$} & \multicolumn{2}{|c|}{ Treatment } & \multirow{2}{*}{$\begin{array}{l}\text { Method \& Definition } \\
\text { of Hyporesponder }\end{array}$} & \multicolumn{2}{|r|}{ Outcome } \\
\hline & & Neuroendovascular & Antiplatelet & & Laboratory & Clinical \\
\hline $\begin{array}{l}\text { Asai et } \\
\quad \text { al., } \\
2016\end{array}$ & $\begin{array}{l}\text { 181: } 189 \\
\text { procedures } \\
(8 / 2010- \\
7 / 2013)\end{array}$ & Coil, stent/coil & $\begin{array}{l}\text { ASA + CLP usually (up } \\
\text { to } 3 \text { antiplatelet drugs } \\
\text { w/ cilostazol), } \geq 5-7 \\
\text { days prior; intrapro- } \\
\text { cedure: heparin (ACT } \\
250-350 \text { sec); post- } \\
\text { stent: IV argatroban } \\
60 \text { mg daily for } 24 \text { hrs }\end{array}$ & $\begin{array}{l}\text { VerifyNow prior } \\
\text { to procedure; } \\
<230 \text { (based } \\
\text { on GRAVITAS } \\
\text { study) }\end{array}$ & $\begin{array}{l}34.9 \% \\
\quad(66 / 189) \\
\text { CLP low } \\
\text { respond- } \\
\text { ers, } 5.8 \% \\
(11 / 189) \\
\text { ASA low } \\
\text { respond- } \\
\text { ers }\end{array}$ & $\begin{array}{l}\text { Overall symptomatic ischemic } \\
\text { events } 4.2 \% \text { ( } 8 / 189) \text {; symp- } \\
\text { tomatic ischemic events in } \\
\text { low responders vs responders } \\
3.0 \%(2 / 66) \text { vs } 4.9 \%(6 / 123), p \\
=0.72 ; \text { new ischemic lesions } \\
\text { on MR-DWI in low responders } \\
\text { vs responders } 66.7 \%(44 / 66) \\
\text { vs } 56.1 \%(69 / 123), p=0.26 ; \\
\text { new ischemic lesions }>5 \text { mm } \\
\text { on MR-DWI in low responders } \\
\text { vs responders } 39.4 \%(26 / 66) \text { vs } \\
21.1 \%(26 / 123), p=0.01\end{array}$ \\
\hline $\begin{array}{c}\text { Brinjikji } \\
\text { et al., } \\
2015\end{array}$ & $\begin{array}{l}\text { 698: } 805 \\
\text { unruptured } \\
\text { aneurysms; } \\
\text { retrospective } \\
\text { review using } \\
\text { International } \\
\text { Retrospec- } \\
\text { tive study } \\
\text { of PED } \\
\text { (IntrePED) } \\
\text { registry } \\
\text { (7/2008- } \\
\text { 2/2013) }\end{array}$ & $\begin{array}{l}\text { PED (ICA, MCA, } \\
\text { PCA, basilar } \\
\text { artery, or other) }\end{array}$ & Not available & $\begin{array}{l}\text { Platelet testing } \\
\text { prior to proce- } \\
\text { dure }(73.2 \% \mathrm{w} / \\
\text { testing) }\end{array}$ & & $\begin{array}{l}\text { Platelet testing group } 5.5 \%(28 / 511) \\
\text { ischemic stroke vs no-platelet } \\
\text { testing group } 0.8 \%(4 / 511) \\
\text { ischemic stroke }(p=0.06) ; \\
\text { platelet testing group } 2.3 \% \\
\text { (12/511) intracranial hemorrhage } \\
\text { vs no-platelet testing group } 0 \\
\text { intracranial hemorrhage }(p= \\
0.04)\end{array}$ \\
\hline $\begin{array}{c}\text { Hwang } \\
\text { et al., } \\
2015\end{array}$ & $\begin{array}{l}228 \\
\text { (5/27/2013- } \\
\text { 4/7/2014; } \\
\text { prospective } \\
\text { randomized } \\
\text { open-label } \\
\text { active-con- } \\
\text { trol trial } \\
\text { w/ blinded } \\
\text { outcome as- } \\
\text { sessment }\end{array}$ & $\begin{array}{l}\text { Coil embolization } \\
\text { (unruptured } \\
\text { aneurysm) }\end{array}$ & $\begin{array}{l}\text { ASA } 100 \text { mg daily (if } \\
\text { HTPR to aspirin, } 300 \\
\text { mg LD), CLP } 75 \text { mg } \\
\text { daily (if HTPR to CLP, } \\
\text { cilostazol } 200 \text { mg LD } \\
\text { w/ } 200 \text { mg daily as } \\
\text { MD if stent-assisted } \\
\text { coiling), } 5 \text { days prior; } \\
\text { antiplatelet therapy } \\
\text { discontinued in coiling } \\
\text { w/o stent; patients } \\
\text { monitored } 24 \text { hrs \& } \\
\text { discharged next day, } \\
\text { follow-up in } 7 \& 30 \\
\text { days after coiling }\end{array}$ & $\begin{array}{l}\text { VerifyNow prior to } \\
\text { procedure; ARU } \\
<550, \text { PRU }<213\end{array}$ & $\begin{array}{l}\text { Hypore- } \\
\text { sponders } \\
\text { randomly } \\
\text { assigned } \\
\text { to stand- } \\
\text { ard or } \\
\text { modified } \\
\text { treatment } \\
\text { groups: } \\
55.3 \% \\
(126 / 228) \text {, } \\
\text { nonhy- } \\
\text { pore- } \\
\text { sponders } \\
44.7 \% \\
(102 / 228)\end{array}$ & $\begin{array}{l}\text { Overall TE events } 3.9 \% \text { (9/228); } \\
\text { TE events in HTPR-standard } \\
\text { vs HTPR-modified treatment vs } \\
\text { non-HTPR group } 11.1 \%(7 / 63) \\
\text { vs } 1.6 \% \text { (1/63) vs } 1 \% \text { (1/102), } \\
\text { p = 0.02; TE w/in } 7 \text { days } 11.1 \% \\
\text { (7/63) standard group vs } 1.0 \% \\
\text { (1/102) non-HTPR group ( } p= \\
0.03 \text { when adjusted for sex, age, } \\
\text { history of CHD, aneurysm diam- } \\
\text { eter, aneurysm neck size); TIMI } \\
\text { bleeding criteria w/in } 30 \text { days } \\
\text { 9.6\% (22/228), } 0 \text { more than mini- } \\
\text { mal TIMI bleeding criteria during } \\
30 \text { days after coil embolization; } \\
\text { 9.5\% (6/63) modified group vs } \\
6.3 \% \text { (4/63) standard group, } p \\
\text { = 0.26; TE events w/in } 30 \text { days } \\
\text { in HTPR-standard vs HTPR- } \\
\text { modified group } 11.1 \% \text { (7/63) vs } \\
1.6 \% \text { (1/63), p = 0.02 }\end{array}$ \\
\hline
\end{tabular}

CONTINUED ON PAGE $898 »$

$50 \%$ within 60 days of the procedure. $5,15,20,36,45$ This is in contrast to much lower complication rates in patients with appropriate clopidogrel response (1\% within 24 hours of the procedure and up to $21.2 \%$ within 30 days of the procedure). ${ }^{5,15,16,20,43,45}$
Several factors influence clopidogrel responsiveness. In multivariate analyses, clopidogrel hyporesponsiveness was associated with age $>55$ years, history of diabetes mellitus, female sex, higher body weight, and body mass index $\left(>25 \mathrm{~kg} / \mathrm{m}^{2}\right) \cdot{ }^{17,33,36,53}$ Some of these risk factors are known 


\section{K. S. Kim et al.}

» CONTINUED FROM PAGE 897

TABLE 2. Outcomes in clopidogrel hyporesponders

\begin{tabular}{|c|c|c|c|c|c|c|}
\hline \multirow{2}{*}{$\begin{array}{l}\text { Authors } \\
\& \text { Year }\end{array}$} & \multirow{2}{*}{$\begin{array}{l}\text { No. of Patients } \\
\text { (study dates)* }\end{array}$} & \multicolumn{2}{|c|}{ Treatment } & \multirow{2}{*}{$\begin{array}{l}\text { Method \& Definition } \\
\text { of Hyporesponder }\end{array}$} & \multicolumn{2}{|r|}{ Outcome } \\
\hline & & Neuroendovascular & Antiplatelet & & Laboratory & Clinical \\
\hline $\begin{array}{l}\text { Nishi et } \\
\text { al., } \\
2016\end{array}$ & $\begin{array}{c}279(5 / 2010- \\
4 / 2013)\end{array}$ & $\begin{array}{l}\text { Stent (carotid, } \\
\text { vertebral), coil } \\
\text { embolization } \\
\text { (unruptured } \\
\text { aneurysm) }\end{array}$ & $\begin{array}{l}\text { ASA } 100 \text { mg + CLP } 75 \\
\text { mg daily, } \geq 5 \text { days } \\
\text { prior, or ASA } 200 \\
\text { mg + CLP } 300 \text { mg } \\
\text { LD; intraprocedure: } \\
\text { IV heparin (ACT } \\
250-300 \mathrm{sec} \text { ) }\end{array}$ & $\begin{array}{l}\text { VerifyNow prior to } \\
\text { procedure }\end{array}$ & $\begin{array}{l}\text { Hyperre- } \\
\text { sponders } \\
30 \%\end{array}$ & $\begin{array}{l}\text { Overall major bleeding events } \\
11.1 \%(31 / 279) \text {; intracranial } \\
\text { hemorrhage } 3.6 \% \text { (10/279), } \\
\text { subarachnoid hemorrhage } 3.2 \% \\
(9 / 279) \text {, cerebral hemorrhage } \\
0.3 \%(1 / 279) ; \text { bleeding events in } \\
\text { hyperresponders vs nonhyper- } \\
\text { responders } 19 \% \text { vs } 7.69 \%\end{array}$ \\
\hline $\begin{array}{c}\text { Tan et al., } \\
2015\end{array}$ & $\begin{array}{c}74(4 / 2011- \\
8 / 2013)\end{array}$ & PED (ACA, PCA) & $\begin{array}{l}\text { ASA } 325 \text { mg daily, CLP } \\
75 \text { mg daily, } 5 \text { days } \\
\text { prior; if emergency, } \\
\text { ASA } 325 \text { mg + } 600 \\
\text { mg CLP LD } 2 \text { hrs } \\
\text { prior to procedure; } \\
\text { nonresponder: CLP } \\
600 \text { mg \&/or ASA } \\
325 \text { mg immediately } \\
\text { postprocedure before } \\
\text { ACT normalization; } \\
\text { intraprocedure: IV } \\
\text { heparin (ACT } 250 \\
\text { sec); abciximab (0.25 } \\
\text { mg/kg LD + } 0.125 \mu \text { g/ } \\
\text { kg/min or } 10 \mu g / m i n \\
\text { for } 12 \text { hrs) if ASA/CLP } \\
\text { hyporesponder }\end{array}$ & $\begin{array}{l}\text { VerifyNow prior to } \\
\text { procedure; ARU } \\
<550, \text { PRU }<230\end{array}$ & $\begin{array}{l}\text { CLP hypore- } \\
\text { sponders } \\
\text { (PRU } \\
>208) \\
52.7 \% \\
(39 / 74)\end{array}$ & $\begin{array}{l}1 \text { day postprocedure: MR-DWI } \\
\text { changes } 39.2 \% \text { (29/74); } 1 \\
\text { wk postprocedure: TE } 6.8 \% \\
\text { (5/74), intracranial hemorrhage } \\
\text { 1.4\% (1/74); } 4 \text { wks postproce- } \\
\text { dure: symptomatic TE in CLP } \\
\text { hyporesponders vs responders } \\
12.8 \%(5 / 39) \text { vs } 0 \% \text {; MR-DWl } \\
\text { changes in CLP hyporesponders } \\
\text { vs responders } 48.4 \% \text { (15/31) vs } \\
53.9 \% \text { (14/26); intracranial hem- } \\
\text { orrhage in hyporesponders vs } \\
\text { responders } 0 \% \text { vs } 2.9 \%(1 / 35)\end{array}$ \\
\hline $\begin{array}{l}\text { Wong } \\
\text { et al., } \\
2015\end{array}$ & $\begin{array}{l}90(5 / 2002- \\
12 / 2012 ; \\
\text { standard } \\
\text { therapy [no } \\
\text { testing] vs } \\
40 \text { tailored } \\
\text { therapy } \\
\text { [testing] })\end{array}$ & $\begin{array}{l}\text { Stent (intracranial } \\
\text { artery stenosis, } \\
\text { aneurysms) }\end{array}$ & $\begin{array}{l}\text { ASA } 325 \text { mg daily, CLP } \\
75 \text { mg daily ( } 300-600 \\
\text { mg LD per MD if } \\
\text { resistant), } 7 \text { days prior }\end{array}$ & $\begin{array}{l}\text { VerifyNow prior \& } \\
\text { postprocedure; } \\
\text { PRU 120-180 }\end{array}$ & $\begin{array}{l}\text { CLP hypore- } \\
\text { sponders } \\
53.1 \% \\
(17 / 32) \\
\text { CLP } \\
\text { hyperre- } \\
\text { sponders } \\
25 \% \\
(8 / 32)\end{array}$ & $\begin{array}{l}\text { Overall TE complications } 1.5 \% \text {; TE } \\
\text { events in CLP hyporesponders } \\
\text { 0; overall hemorrhagic complica- } \\
\text { tions } 16.9 \% \text {; bleeding events in } \\
\text { CLP hyperresponders } 25 \% \text {; TE } \\
\text { events during the procedure in } \\
\text { standard vs tailored group } 5.6 \% \\
\text { (5/90) vs } 2.5 \%(1 / 40), p=0.648 ; \\
\text { TE events after procedure in } \\
\text { standard vs tailored group } 1.1 \% \\
\text { (1/90) vs } 2.5 \%(1 / 40), p=0.522 ; \\
\text { TIMI bleeding events in standard } \\
\text { vs tailored group } 17.8 \%(16 / 90) \\
\text { vs } 15.0 \%(6 / 40), p=0.455\end{array}$ \\
\hline
\end{tabular}

$\mathrm{ACA}=$ anterior cerebral artery; $\mathrm{ACT}=$ activated clotting time; $\mathrm{aPTT}=$ activated partial thromboplastin time; $\mathrm{ASA}=$ acetylsalicylic acid (aspirin); $\mathrm{CHD}=$ coronary heart disease; $\mathrm{CLP}=$ clopidogrel; ICA = internal carotid artery; ICH = intracerebral hemorrhage; IV = intravenous; $L D=$ loading dose; $M C A=$ middle cerebral artery; $M D=$ maintenance dose; $\mathrm{MPA}=$ maximum platelet aggregation; $\mathrm{NS}=$ not significant; $\mathrm{PCA}=$ posterior cerebral artery; $\mathrm{TIA}=$ transient ischemic attack; $\mathrm{TIMI}=\mathrm{Thrombolysis}$ in Myocardial Infarction; $\mathrm{Tx}=$ treatment.

* Dates given as month/year or month/day/year.

† TIMI bleeding criteria: major = any intracranial bleeding, clinically overt signs of hemorrhage associated with a drop in hemoglobin of $\geq 5 \mathrm{~g} / \mathrm{dl}$, fatal bleeding; minor = clinically overt bleeding, resulting in hemoglobin drop of 3 to $<5 \mathrm{~g} / \mathrm{dl}$.

to increase platelet reactivity independent of antiplatelet therapy and are of clinical importance when predicting thromboembolic events. However, clopidogrel hyporesponsiveness did not consistently translate to thromboembolic events (stent thrombosis, restenosis, or any clinical outcome) in past studies. ${ }^{5,33,53}$ Interestingly, Prabhakaran et al. found no linear association between preprocedural weekly clopidogrel dose and P2Y12 percentage inhibition. ${ }^{53}$ Other factors associated with thromboembolic events include history of hypertension, posterior location of treatment, longer operation time, being a current smoker, larger aneurysm size, aneurysm neck diameter, multiple 
TABLE 3. Summary of antiplatelet agents used for neuroendovascular indications

\begin{tabular}{|c|c|c|c|c|c|c|c|c|c|}
\hline \multirow[b]{2}{*}{ Variable } & \multicolumn{9}{|c|}{ Antiplatelet Agent } \\
\hline & Aspirin & Clopidogrel & Ticagrelor & Prasugrel & Cilostazol & Cangrelor & Abciximab & Eptifibatide & Tirofiban \\
\hline $\begin{array}{c}\text { Mechanism } \\
\text { of action }\end{array}$ & $\begin{array}{l}\text { Irreversible } \\
\text { inhibitor } \\
\text { cyclooxy- } \\
\text { genases } \\
1 \& 2\end{array}$ & $\begin{array}{l}\text { Irreversible } \\
\text { inhibitor } \\
\text { P2Y12 } \\
\text { ADP } \\
\text { receptor }\end{array}$ & $\begin{array}{c}\text { Reversible } \\
\text { inhibitor } \\
\text { P2Y12 } \\
\text { ADP } \\
\text { receptor }\end{array}$ & $\begin{array}{l}\text { Irreversible } \\
\text { inhibitor } \\
\text { P2Y12 } \\
\text { ADP } \\
\text { receptor }\end{array}$ & $\begin{array}{l}\text { Reversible } \\
\text { phosphodi- } \\
\text { esterase in- } \\
\text { hibitor, ADP } \\
\text { inhibitor }\end{array}$ & $\begin{array}{l}\text { Reversible } \\
\text { inhibitor } \\
\text { P2Y12 ADP } \\
\text { receptor }\end{array}$ & $\begin{array}{l}\text { Irreversible } \\
\text { inhibitor } \\
\text { glycopro- } \\
\text { tein Ilb/Illa } \\
\text { receptor }\end{array}$ & $\begin{array}{l}\text { Reversible } \\
\text { inhibitor } \\
\text { glycopro- } \\
\text { tein Ilb/Illa } \\
\text { receptor }\end{array}$ & $\begin{array}{l}\text { Reversible } \\
\text { inhibitor } \\
\text { glycopro- } \\
\text { tein Ilb/llla } \\
\text { receptor }\end{array}$ \\
\hline Onset & $7-60$ mins & $2 \mathrm{hrs}$ & 30 mins & $15-30$ mins & $3-6 \mathrm{hrs}$ & 2 mins & $2 \mathrm{hrs}$ & Immediate & 5 mins \\
\hline $\begin{array}{l}\text { Duration of } \\
\text { action }\end{array}$ & $3-5$ days & $3-7$ days & $12-24 \mathrm{hrs}$ & $5-9$ days & $48 \mathrm{hrs}$ & $1 \mathrm{hr}$ & $48 \mathrm{hrs}$ & $2-4 \mathrm{hrs}$ & $3-8 \mathrm{hrs}$ \\
\hline Metabolism & Minimal & $\begin{array}{c}\text { CYP450 } \\
2 \text { C9 }\end{array}$ & $\begin{array}{c}\text { CYP450 } \\
3 \mathrm{~A} 4\end{array}$ & $\begin{array}{l}\text { CYP450 } \\
3 \mathrm{~A} / 2 \mathrm{~B} 6\end{array}$ & Minimal & $\begin{array}{c}\text { Dephosphoryla- } \\
\text { tion in serum }\end{array}$ & None & Minimal & Minimal \\
\hline
\end{tabular}

PED placements, and lack of statin use..$^{5,16,20,26,67}$ Both clinical and procedure-related factors must be considered before determining a patient's risk status for HTPR.

Numerous reports of platelet function testing for clopidogrel have been published in an effort to define the role of HTPR on thromboembolic and bleeding events after neuroendovascular stenting and coiling (Table 1). Assimilation of all these reports is not particularly straightforward because of the variety of procedures, doses, methods of platelet function testing, and time to follow-up in each publication. For instance, procedures such as deployment of the PED may be associated with a higher thrombosis rate due to the technical aspects and the risk factors of the patients who receive this device, particularly when compared with carotid stenting, where flow in the target vessel is higher and less tortuous. Cases of stent-assisted aneurysm coiling typically have a higher risk of thrombosis than aneurysm coiling alone.

Several studies have reviewed the impact of clopidogrel hyporesponsiveness in patients undergoing PED deployment. Delgado Almandoz et al. used $75 \mathrm{mg}$ of clopidogrel and $325 \mathrm{mg}$ of aspirin daily in 44 patients with PEDs. ${ }^{16}$ They demonstrated a significant association with clopidogrel hyporesponsiveness (PRU > 240) and thromboembolic events. On the opposite end of the spectrum, they also demonstrated a significant association with $\mathrm{PRU}<60$ and bleeding events. Daou et al. used $75 \mathrm{mg}$ of clopidogrel and $325 \mathrm{mg}$ of aspirin daily (started 10 days prior to the procedure) in 231 patients with PEDs. ${ }^{14}$ Higher odds of complications were associated with clopidogrel PRU values $<70$ (hemorrhagic) and $>150$ (thromboembolic) after controlling for aneurysm size and aspirin treatment (OR 3, 95\% CI 1.2-7.5). As a result, many current clinical trials of flow-diverting treatments for aneurysms have specific parameters for platelet responsiveness in their inclusion criteria (clinical trial no. NCT02186561; clinicaltrials.gov).

Other studies using platelet function testing in patients receiving PEDs were not as definitive. Some investigators found no difference in complications in patients with HTPR. ${ }^{26}$ Oran et al. used the Multiplate analyzer in 1 cohort of patients and after comparing them with a cohort that did not receive testing, they suggested that thromboembolic complications and morbidity (but not bleeding) were higher in the nontested group. ${ }^{46}$ Another study inves- tigated patients receiving clopidogrel prior to PEDs and divided the population into 2 cohorts: patients with PRU < 208 and those with PRU $>208 .{ }^{67}$ No difference in bleeding rates or ischemic lesions based on MR diffusion-weighted imaging (DWI) were evident, although there were higher overall thromboembolic events noted in patients with PRU $>208(12.9 \%$ vs $0 \%$ in PRU $<208$ group, $\mathrm{p}=0.06)$. One confounding factor in this study was the concomitant use of abciximab throughout and after the stent deployment procedures (and for 12 hours total).

Clopidogrel hyporesponsiveness has also been investigated in other neuroendovascular procedures and has yielded similar results. Patients undergoing elective unruptured aneurysm coiling were initiated on clopidogrel and other antiplatelet agents at least 5 days prior to their procedure or with a loading dose the day prior to the procedure in 4 separate studies. The results of these reports are relatively consistent. Three of the studies demonstrated that an increased PRU was associated with thromboembolic events. ${ }^{15,28,33}$ Asai et al. did not see a difference in symptomatic thromboembolic events, though MR-DWI lesions were increased in patients with HTPR. ${ }^{5}$ Several other studies reported the results of a mix of patients undergoing aneurysm coiling plus stent-assisted coiling, carotid stenting, or cerebral angioplasty. One study demonstrated a significant difference in mortality in patients with clopidogrel HTPR compared with those with target PRU (23\% vs $4 \%$ target PRU, $p=0.03$ ), but no association with thromboembolic complications between cohorts. ${ }^{45}$ Fifi and colleagues evaluated clopidogrel response in patients undergoing aneurysm coiling or carotid stenting and suggested that occurrence of stent thrombosis was frequently associated with HTPR. ${ }^{20}$ Conversely, Koerner et al. described patients undergoing stent procedures to various arteries (carotid, vertebral, or basilar) and demonstrated no increase in thromboembolic events in patients with HTPR. ${ }^{35}$ Two other studies including patients receiving aneurysm coiling (with or without stent assist) suggested that a low PRU in patients receiving clopidogrel was associated with an increased rate of bleeding events (incidence of thromboembolic events was not reported). ${ }^{22,44}$

The most commonly used method to overcome clopidogrel hyporesponsiveness was to administer additional loading doses of clopidogrel prior to the procedure. Lee 
et al. reported a nonstatistical reduction in PRU before and after an additional 300-mg clopidogrel loading dose, but this was based on a small number of patients. ${ }^{36} \mathrm{On}$ the other hand, Fifi et al. reported a significant increase in P2Y12 percentage inhibition in clopidogrel nonresponders after an additional 150-600 mg clopidogrel loading dose and observed a significant percentage of patients converting to responders. ${ }^{20}$ Alternative P2Y12 inhibitors may be considered in this situation as well.

On the opposite end of the spectrum, there are few studies describing the incidence of bleeding events in clopidogrel hyperresponders. Clopidogrel hyperresponsiveness occurs in $14 \%-30 \%$ of patients and has been associated with major and minor bleeding events based on various laboratory definitions. ${ }^{15,16,22,34,44}$ Nishi et al. identified PRU $\leq 175$ as the optimal cutoff. Clopidogrel hyperresponders were at a 2.8 times higher risk for major and minor bleeding events than nonhyperresponders. ${ }^{44}$ The main difference between the 2 groups was a higher incidence in puncture site hemorrhage and not intracranial hemorrhage or retroperitoneal hemorrhage. Goh et al. and Kashiwazaki et al. found $\geq 72-74 \mathrm{P} 2 \mathrm{Y} 12$ percentage inhibition as the optimal cutoff for predicting major bleeding events. ${ }^{22,34}$ Lower ARU values were not associated with bleeding events. ${ }^{34,44}$

Not all patients who exhibit hyperresponsiveness consistently express this phenotype after neuroendovascular intervention. Within 6 months of the endovascular procedure, $77.3 \%$ of patients who were clopidogrel responders became hyperresponsive and $41.2 \%$ of these patients experienced major and minor bleeding events. ${ }^{15}$ In clopidogrel hyperresponders, the associated major and minor bleeding events were $19 \%$ within 1 week of the procedure, $15.4 \%-$ $44.4 \%$ within 30 days of the procedure, and $33.3 \%$ within 6 months of the procedure. ${ }^{15,16,34,44}$ Comparatively, major and minor bleeding events in clopidogrel nonhyperresponders were significantly lower at $7.69 \%$ within 1 week of the procedure, $5.7 \%-6.9 \%$ within 30 days of the procedure, and $2.6 \%$ within 6 months of the procedure..$^{15,16,34,44}$ Intracranial hemorrhage occurred in both clopidogrel hyperresponders and nonhyperresponders. ${ }^{15,16,44}$

Definitive conclusions are difficult to make regarding clopidogrel response and treatment success in neuroendovascular procedures due to the heterogeneity of the procedures, cutoff values used with platelet function testing, clopidogrel dosing, and the use of alternative antiplatelet agents. The majority of the literature evaluating concomitant clopidogrel and aspirin use seems to suggest that HTPR is at least modestly associated with thromboembolic events. Platelet function testing seems advisable for detection of risk of thrombosis based on patient characteristics, difficulty of the procedure, and number of stents deployed. ${ }^{26,67} \mathrm{An}$ increase in bleeding rates in patients with LTPR has also been reported in some studies, although this is less well defined. ${ }^{15}$

\section{P2Y12 Inhibitors}

\section{Prasugrel and Ticagrelor}

Agents that act at the P2Y12 receptor, but exhibit considerably less variability in response, include prasugrel and ticagrelor. Both agents have been used as alternatives to clopidogrel in patients with HTPR with some success
(Table 4). Patients receiving a PED who exhibited HTPR to clopidogrel were switched to prasugrel in one study. No thromboembolic or bleeding complications occurred. ${ }^{13}$ Similarly, 3 separate cohorts of clopidogrel hyporesponsive patients undergoing a mix of neuroendovascular procedures were switched from clopidogrel to prasugrel or ticagrelor. Significantly higher hemorrhagic events were noted with prasugrel therapy in 1 study $(\mathrm{p}=0.02)$, but this was not significant when a patient with basilar artery perforation was excluded from the data analysis $(\mathrm{p}=$ $0.09) .{ }^{2}$ Only 1 bleeding complication (prasugrel) occurred in the other 2 cohorts. ${ }^{25,66}$ No thromboembolic complications were noted in any one of these 3 studies. These preliminary data suggest that using alternatives to clopidogrel in patients with HTPR may be reasonable, as more patients will become responsive to clopidogrel alternative therapies. However, these alternatives must be used with caution as prasugrel and ticagrelor may convert hyporesponders to hyperresponders, and this has been associated with higher major and minor bleeding rates in both PCI and neuroendovascular settings. ${ }^{6,15,51}$ Similar findings of high bleeding rates in clopidogrel hyperresponders have also been observed, and thus further platelet function testing may be necessary in patients whose antiplatelet therapy is modified. There are limited clinical outcomes data with ticagrelor therapy, and it should be used with caution as the currently approved FDA dosing regimen $(180 \mathrm{mg}$ loading/90 mg twice daily) may produce PRUs $<40.3,4,24$

\section{Cilostazol}

Hwang et al. published a prospective study of 228 patients undergoing elective aneurysm coil embolization (with the majority having a stent assist). ${ }^{28}$ Three cohorts of patients were compared: those with non-HTPR on clopidogrel (standard regimen: clopidogrel $75 \mathrm{mg}$ daily, aspirin $100 \mathrm{mg}$ daily), those with HTPR who received the standard treatment regimen, and those with HTPR who received a modified regimen (clopidogrel $75 \mathrm{mg}$ daily, aspirin $300 \mathrm{mg}$ daily, and cilostazol $200 \mathrm{mg}$ loading dose, then $200 \mathrm{mg}$ daily as maintenance dose if stent-assisted coiling). Thromboembolic events were lower in patients with the modified regimen than in patients with HTPR who received the standard regimen $(11.1 \%$ standard vs $1.6 \%$ modified, $\mathrm{p}=0.02$ ). No differences in bleeding complications were observed among the 3 cohorts. Thus, it appears that the addition of cilostazol to the combination of aspirin and clopidogrel in patients with HTPR may be an acceptable strategy to overcome clopidogrel hyporesponsiveness.

\section{Glycoprotein IIb/llla Inhibitors}

Glycoprotein IIb/IIIa inhibitors may be used during neuroendovascular procedures, usually either as an adjunct to DAT or as rescue therapy for acute thrombosis. Intravenous administration of glycoprotein IIb/IIIa inhibitors may be used in patients undergoing aneurysm coil embolization or stent placement as an additive protective measure for procedural thrombosis (analogous to their typical use in PCI). ${ }^{62}$ One prospective study compared patients who received preprocedure antiplatelet therapy (aspirin and clopidogrel) versus those who only received 
TABLE 4. Alternatives to clopidogrel in patients with HTPR

\begin{tabular}{|c|c|c|c|c|c|c|}
\hline \multirow{2}{*}{$\begin{array}{l}\text { Authors } \\
\text { \& Year }\end{array}$} & \multirow{2}{*}{$\begin{array}{l}\text { No. of Patients } \\
\text { (study dates) }\end{array}$} & \multicolumn{2}{|r|}{ Treatment } & \multirow{2}{*}{$\begin{array}{l}\text { Method \& } \\
\text { Definition }\end{array}$} & \multicolumn{2}{|r|}{ Outcome } \\
\hline & & Neuroendovascular & Antiplatelet & & Laboratory & Clinical \\
\hline $\begin{array}{c}\text { Akbari } \\
\text { et al., } \\
2013\end{array}$ & $\begin{array}{l}55 \text { ASA/CLP } \\
\text { vs } 31 \text { ASA/ } \\
\text { prasugrel } \\
(2 / 15 / 2010- \\
10 / 31 / 2011)\end{array}$ & $\begin{array}{l}\text { Coil, PED, stent } \\
\text { (intracranial an- } \\
\text { eurysms, AVM, } \\
\text { dural arterio- } \\
\text { venous fistula, } \\
\text { intra-/extracra- } \\
\text { nial stenosis) }\end{array}$ & $\begin{array}{l}\text { ASA } 325 \text { mg daily + CLP } 75 \text { mg } \\
\text { daily (CLP change to prasugrel } \\
60 \text { mg LD/10 mg daily), } \geq 7 \text { days } \\
\text { prior; intraprocedure: IV heparin } \\
\text { (ACT } 2 \text { times baseline) }\end{array}$ & $\begin{array}{l}\text { VerifyNow } \\
\text { prior to } \\
\text { procedure } \\
\text { \& as } \\
\text { needed, } \\
\text { P2Y12\% } \\
\text { inhibition } \\
>40 \%\end{array}$ & & $\begin{array}{l}\text { Overall hemorrhagic events } \\
9.3 \% \text { (8/86); hemorrhagic } \\
\text { events ASA/CLP vs ASA/ } \\
\text { prasugrel } 3.6 \% \text { vs } 19.4 \% \text {, } \\
p=0.02 ; \text { intracranial } \\
\text { hemorrhagic events ASA/ } \\
\text { CLP vs ASA/prasugrel } \\
1.8 \% \text { vs } 12.9 \%\end{array}$ \\
\hline $\begin{array}{l}\text { Chalouhi } \\
\text { et al., } \\
2013\end{array}$ & $\begin{array}{c}7(11 / 2011- \\
7 / 2012)\end{array}$ & $\begin{array}{r}\text { Posterior circula- } \\
\text { tion aneurysm }\end{array}$ & $\begin{array}{l}\text { ASA } 81 \text { mg daily, CLP } 75 \text { mg daily } \\
\text { (CLP poor responder: prasugrel } \\
40 \text { mg LD, } 5 \text { mg MD), } 10 \text { days } \\
\text { prior; intraprocedure: heparin } \\
100 \text { units/kg bolus, then dosed } \\
\text { per ACT } 2 \text { times baseline }\end{array}$ & $\begin{array}{l}\text { VerifyNow } \\
\text { prior to } \\
\text { procedure, } \\
\text { P2Y12 \% } \\
\text { inhibition } \\
\geq 30 \%\end{array}$ & & $\begin{array}{l}\text { Mean } 5.5 \text { mos (range } 3-7 \\
\text { mos): no ischemic or } \\
\text { bleeding complications }\end{array}$ \\
\hline $\begin{array}{l}\text { Stetler } \\
\text { et al., } \\
2013\end{array}$ & $\begin{array}{l}\text { 16: prasugrel } \\
(1 / 2009- \\
7 / 2011)\end{array}$ & $\begin{array}{l}\text { Stent (intracranial } \\
\text { aneurysm, intra- } \\
\text { cranial stenosis, } \\
\text { extracranial } \\
\text { artery pathol- } \\
\text { ogy) }\end{array}$ & $\begin{array}{l}\text { CLP } 75 \mathrm{mg} \text { daily (CLP } 300 \mathrm{mg} \text { LD, } \\
\text { if urgent; CLP nonresponders } \\
\text { changed to prasugrel } 40 \mathrm{mg} \\
\text { LD, then } 5-10 \mathrm{mg} \text { daily), ASA } \\
\geq 81 \mathrm{mg} \text { daily, } \geq 7 \text { days prior; } \\
\text { intraprocedure: IV heparin (ACT } \\
250-300 \mathrm{sec} \text { ); postprocedure: } \\
\text { heparin } 500 \text { units } / \mathrm{hr}+\text { dextran } \\
4020 \mathrm{ml} / \mathrm{hr} \text { when PTT }<100 \text {, for } \\
12-18 \mathrm{hrs}\end{array}$ & $\begin{array}{l}\text { VerifyNow } \\
\text { prior to } \\
\text { procedure; } \\
\text { ARU } \\
350-550 \\
\text { P2Y12\% } \\
\text { inhibition } \\
>20 \%\end{array}$ & $\begin{array}{l}\text { P2Y12 \% inhibition } \\
\text { median } 2 \% \text { \& } \\
\text { increased to } \\
>50 \% \text { in } 77 \% \\
\text { of patients w/ } \\
\text { prasugrel }\end{array}$ & $\begin{array}{l}\text { Mean } 3 \text { mos (range } 1 \text { to }>6 \\
\text { mos): intracranial bleed- } \\
\text { ing or ischemic complica- } \\
\text { tions } 0 \text {, retroperitoneal } \\
\text { hematoma } 1\end{array}$ \\
\hline $\begin{array}{l}\text { Hanel } \\
\text { et al., } \\
2014\end{array}$ & $\begin{array}{c}18(10 / 2011- \\
8 / 2012)\end{array}$ & $\begin{array}{l}\text { Stent, coil, PED } \\
\text { (intra-/extra- } \\
\text { cranial artery; } \\
16 \text { elective, } 2 \\
\text { emergency) }\end{array}$ & $\begin{array}{l}\text { ASA } 650 \text { mg LD (CLP } 600 \text { mg LD } \\
\text { + ASA } 650 \text { mg LD, if semiur- } \\
\text { gent), then } 325 \text { mg daily + CLP } \\
300 \text { mg LD (if CLP nonre- } \\
\text { sponder, changed to ticagrelor } \\
180 \text { mg LD, then } 75 \text { mg daily), } 7 \\
\text { days prior }\end{array}$ & $\begin{array}{l}\text { VerifyNow } \\
\text { prior to } \\
\text { procedure; } \\
\text { P2Y12 \% } \\
\text { inhibition } \\
>30 \%\end{array}$ & $\begin{array}{l}\text { P2Y12 \% inhibition } \\
<20 \% \text { increased } \\
\text { to P2Y12 \% } \\
\text { inhibition }>60 \% \\
\text { in } 94.4 \% \text { of } \\
\text { patients w/ } \\
\text { ticagrelor }\end{array}$ & $\begin{array}{l}\text { No adverse effects during } \\
\text { or following surgery }\end{array}$ \\
\hline
\end{tabular}

$\mathrm{AVM}=$ arteriovenous malformation

intraprocedural intraarterial abciximab prior to elective aneurysm coiling. ${ }^{37}$ Patients who received only intraarterial abciximab had no difference in thromboembolic or bleeding complications compared with those who took DAT in the days prior to the procedure, suggesting intraarterial glycoprotein IIb/IIIa inhibitor infusion may be a suitable replacement for DAT during the procedure. Similarly, glycoprotein IIb/IIIa inhibitors have been used in patients who demonstrate HTPR during neuroendovascular procedures as a means of preventing acute thrombosis in high-risk patients. Data supporting the use of glycoprotein IIb/IIIa inhibitors as the sole agent during neuroendovascular interventions are not sufficient to merit doing this as a standard practice, but the available data do suggest that it may be a reasonable adjunct if it is not possible to load antiplatelet agents prior to the procedure or if patients are known to have HTPR during the procedure.

Glycoprotein IIb/IIIa inhibitors are also used as rescue therapy when thrombosis occurs during the procedure. Typically, procedural thromboses are detected quickly and can be addressed before substantial propagation. Therefore, the thrombosis is not mature and fibrin-rich; rather, the thrombosis is usually the initial strands of platelets aggregating after initial activation that is amenable to dispersion with glycoprotein IIb/IIIa inhibitors. Numerous reports of using intraarterial bolus infusions of glycoprotein IIb/IIIa inhibitors for successful stent or coil thrombosis treatment are available in the literature. ${ }^{23,29,31,50,57,58}$ Clinicians may also follow the intraarterial bolus with an infusion (again, based on PCI dosing) to run for at least 12 hours after the procedure to further protect against acute thrombosis. ${ }^{1,18}$ Glycoprotein IIb/IIIa inhibitors also appear to be as effective as and safer than intraarterial thrombolysis, further cementing their role in rescue therapy.

The optimal choice of glycoprotein IIb/IIIa inhibitor is not clear and may depend on the formulary preference within each individual institution. No prospective studies in the neuroendovascular realm have been conducted to identify the best agent, and all of the readily available options (abciximab, eptifibatide, tirofiban) have data to support their use in this clinical setting. Glycoprotein IIb/IIIa inhibitors differ in pharmacokinetics, and these differences may be important as they pertain to antiplatelet action during and after the procedure. Briefly, abciximab tends 


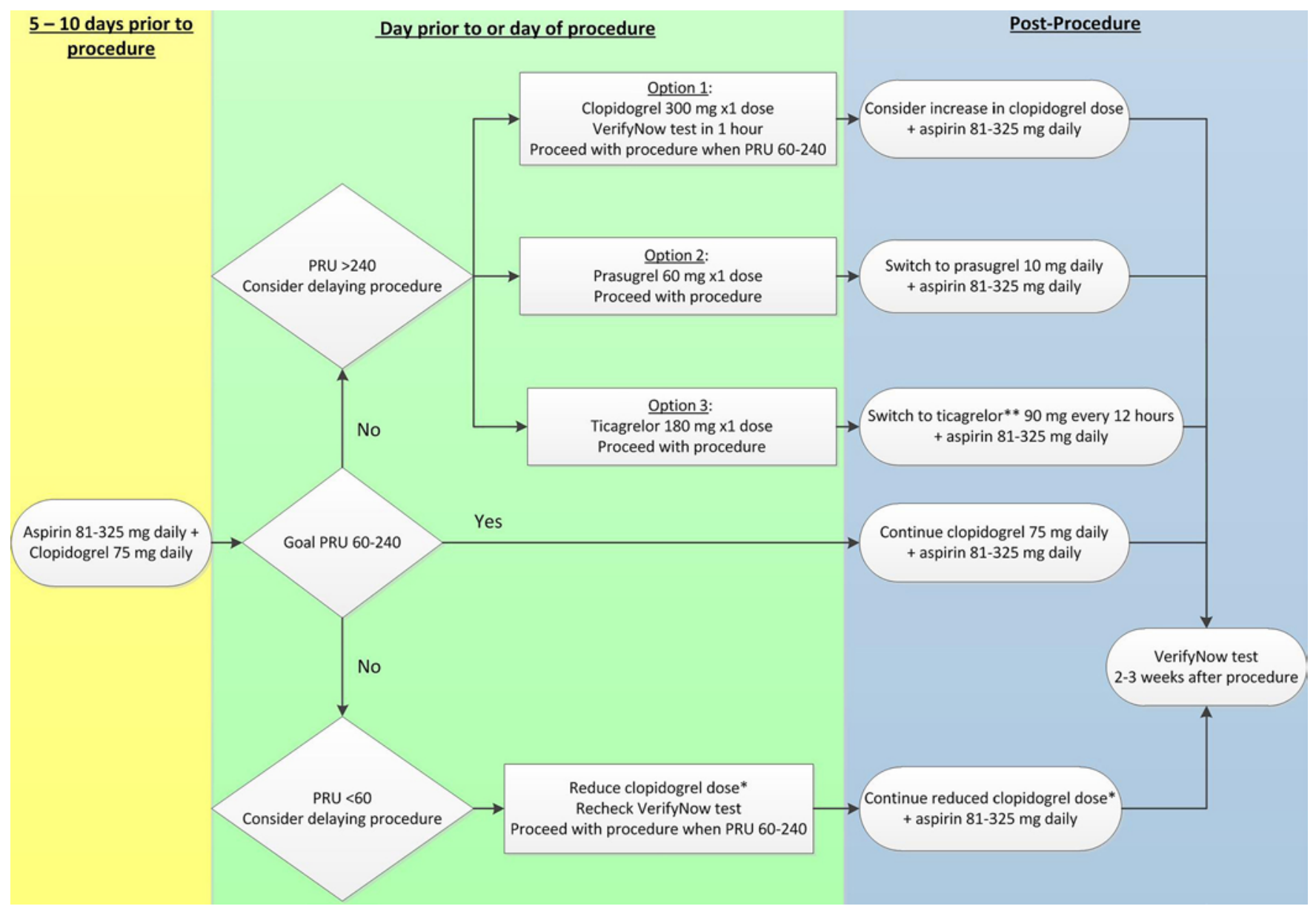

FIG. 1. Protocol for clopidogrel responsiveness using the VerifyNow test in patients undergoing neuroendovascular procedures. When possible, patients undergoing neuroendovascular stenting should be initiated on DAT 5-7 days prior to the procedure. For select patients and procedures, VerifyNow platelet function analysis should be evaluated prior to the procedure. For patients with PRUs > 240 (HTPR), several potential options exist for rapidly gaining platelet inhibition (options 1-3), each with a corresponding postprocedure treatment plan. For patients with PRUs $<60$ (LTPR), clinicians may consider reducing the clopidogrel dose prior to the procedure. Individuals who are hyperresponders to clopidogrel may require a reduced dose or an alternative agent. Patients with a PRU within the goal of 60-240 should continue DAT as initially prescribed. The optimal range of platelet reactivity is not well defined; some clinicians may elect to use a lower threshold of platelet reactivity, such as $60-150.14$ * $C$ lopidogrel dose reduction may be to $75 \mathrm{mg}$ every other day or every third day. The prescriber can maintain the patient on this dose for 7-14 days, then recheck VerifyNow. If PRU remains $<60$, consider reducing the dose to $75 \mathrm{mg}$ weekly or $5 \mathrm{mg}$ (oral solution) per day. Reevaluate VerifyNow 5-7 days after each dose change. May also consider using ticagrelor in patients in whom PRU remains $<60$ despite significant clopidogrel dose reduction. ${ }^{*}$ The FDA recommends decreasing the aspirin dose to $81 \mathrm{mg}$ daily in patients on ticagrelor therapy based on post hoc analysis completed in the Platelet Inhibition and Patient Outcomes (PLATO) trial, where it showed paradoxical worsening of primary efficacy outcomes (composite of death from vascular causes, myocardial infarction, or stroke at 12 months) with an aspirin dose $\geq 300 \mathrm{mg}$ daily. This may be due to an aspirin and ticagrelor interaction and their effect on reduced platelet reactivity. ${ }^{39}$ The effects of an aspirin dose $\geq 300 \mathrm{mg}$ daily with ticagrelor therapy is currently unknown in patients undergoing neuroendovascular procedures, and therefore close monitoring is advised. Figure is available in color online only.

to have a longer action on platelets due to the irreversible binding on the glycoprotein IIb/IIIa receptor. As a result, an intraarterial bolus of abciximab is likely to have a more prolonged pharmacological effect than other glycoprotein IIb/IIIa inhibitors. In addition, in the instance in which bleeding complications ensue, platelet transfusion is likely to be more effective in restoring hemostatic balance in patients receiving abciximab due to the irreversible binding. Other glycoprotein IIb/IIIa inhibitors, such as eptifibatide, exhibit reversible binding and may inhibit recently transfused platelets in the bleeding patient. ${ }^{40,65}$

\section{Management of DAT for Neuroendovascular Procedures}

Platelet function testing appears advisable prior to neuroendovascular procedures, particularly in patients with risk factors for variable clopidogrel response and cases associated with complex aneurysm coil embolization, flow diversion devices, or intracranial stenting. Within each institution, consistent use of a well-validated platelet function test may aid prescribers in predicting clopidogrel responsiveness. The utility of these tests for aspirin resistance is of questionable significance in the majority of these patients. Protocols for adjusting clopidogrel therapy based on plate- 
let responsiveness have been proposed, but not validated at this point (Fig. 1). ${ }^{16}$ Of note, the VerifyNow PRU cutoff value of 60-240 was based on a study by Delgado Almandoz; however, based on PCI literature, the VerifyNow PRU cutoff value is 85-208. There has been no comparison study to validate a PRU cutoff of 85-208 in the neuroendovascular setting, but many of the cutoff values used in neuroendovascular studies were adapted or extrapolated from initial PCI literature.

When possible, patients should be initiated on clopidogrel and aspirin at least 5 days prior to the procedure to permit these agents to reach maximum pharmacological effect. However, many practitioners start patients on the regimen 14 days prior to the procedure. Patients taking clopidogrel prior to the procedure (compared with loading immediately prior to the procedure) may have lower thromboembolic complications. ${ }^{33}$ Patient noncompliance is a major reason for measured HTPR, so specific emphasis to the patient on the importance of these therapies is prudent. If patients present with an urgent need for neuroendovascular intervention, a loading dose of clopidogrel (typically 300-600 mg) is necessary and can be administered orally or via a nasogastric tube..$^{20,45,46,67}$ Likewise, a loading dose may be reasonable if patients present prior to the procedure with PRU > 240 despite preprocedural DAT. This accounts for patients who failed to be adherent to the preprocedure clopidogrel regimen, as well as any patients that merely require a larger loading dose to obtain a therapeutic effect.

For patients who exhibit HTPR with clopidogrel, there are 3 primary ways to overcome clopidogrel hyporesponsiveness: 1) administer an additional clopidogrel loading dose (300 or $600 \mathrm{mg}$ ), 2) change clopidogrel to prasugrel, or 3 ) change clopidogrel to ticagrelor. The safety profile of prasugrel or ticagrelor is uncertain for neurosurgical patients due to the limited availability of data, although it is known that these agents will more predictably provide a high percentage of P2Y12 inhibition and further decrease PRU. 2-4,16,24,25,37

In patients who have exquisite response to clopidogrel and have PRU $<60$, lower doses of clopidogrel may be necessary. A specific dose-response model has not been developed for hypermetabolizers of clopidogrel, so clinicians are often left to empirical dose reduction and subsequent evaluation of the dose (ideally after 7-14 days on a reduced dose). This usually takes longer than the 5-7 days for hypometabolizers because the extensive activation of clopidogrel prolongs the extent of pharmacological effect. Continuing to give a clopidogrel dose of $75 \mathrm{mg}$ is convenient for the patient because of the available dosage forms, i.e., the patient need only to take the dose less frequently (e.g., every other day or every 3 days). Doses as low as 5 mg daily may be advisable for some hypermetabolizers to avoid giving essentially a week's worth of clopidogrel all at the same time. A recipe for a stable oral suspension is available and easy to make. ${ }^{64}$

\section{Summary}

Combination antiplatelet therapy during neuroendovascular procedures, particularly intracranial artery stenting, is a mainstay in therapy. Current evidence suggests that clopidogrel and aspirin are the preferred combination for this therapy. The pharmacogenetic variability associated with clopidogrel response may need to be considered in cases in which the risk of thrombosis is more substantial (e.g., PEDs). Routine platelet function testing may be helpful in identifying patients at risk for HTPR, and the use of alternative antiplatelet agents such as prasugrel, ticagrelor, or cilostazol may be necessary. In addition, with alteration in platelet therapy, combined planned, long-term platelet function testing is advised in order to identify patients with LTPR.

\section{References}

1. Aggour M, Pierot L, Kadziolka K, Gomis P, Graftieaux JP: Abciximab treatment modalities for thromboembolic events related to aneurysm coiling. Neurosurgery 67 (2 Suppl Operative):503-508, 2010

2. Akbari SH, Reynolds MR, Kadkhodayan Y, Cross DT III, Moran CJ: Hemorrhagic complications after prasugrel (Effient) therapy for vascular neurointerventional procedures. J Neurointerv Surg 5:337-343, 2013

3. Alexopoulos D, Galati A, Xanthopoulou I, Mavronasiou E, Kassimis G, Theodoropoulos KC, et al: Ticagrelor versus prasugrel in acute coronary syndrome patients with high on-clopidogrel platelet reactivity following percutaneous coronary intervention: a pharmacodynamic study. J Am Coll Cardiol 60:193-199, 2012

4. Alexopoulos D, Xanthopoulou I, Gkizas V, Kassimis G, Theodoropoulos KC, Makris G, et al: Randomized assessment of ticagrelor versus prasugrel antiplatelet effects in patients with ST-segment-elevation myocardial infarction. Circ Cardiovasc Interv 5:797-804, 2012

5. Asai T, Miyachi S, Izumi T, Matsubara N, Haraguchi K, Yamanouchi T, et al: Relationship between low response to clopidogrel and periprocedural ischemic events with coil embolization for intracranial aneurysms. J Neurointerv Surg 8:752-755, 2016

6. Bonello L, Mancini J, Pansieri M, Maillard L, Rossi P, Collet F, et al: Relationship between post-treatment platelet reactivity and ischemic and bleeding events at 1-year follow-up in patients receiving prasugrel. J Thromb Haemost 10:19992005, 2012

7. Bonello L, Tantry US, Marcucci R, Blindt R, Angiolillo DJ, Becker R, et al: Consensus and future directions on the definition of high on-treatment platelet reactivity to adenosine diphosphate. J Am Coll Cardiol 56:919-933, 2010

8. Breet NJ, van Werkum JW, Bouman HJ, Kelder JC, Ruven HJ, Bal ET, et al: Comparison of platelet function tests in predicting clinical outcome in patients undergoing coronary stent implantation. JAMA 303:754-762, 2010

9. Brinjikji W, Lanzino G, Cloft HJ, Siddiqui AH, Hanel RA, Kallmes DF: Platelet testing is associated with worse clinical outcomes for patients treated with the Pipeline embolization device. AJNR Am J Neuroradiol 36:2090-2095, 2015

10. Byrne JV, Hope JK, Hubbard N, Morris JH: The nature of thrombosis induced by platinum and tungsten coils in saccular aneurysms. AJNR Am J Neuroradiol 18:29-33, 1997

11. Castro E, Fortea F, Villoria F, Lacruz C, Ferreras B, Carrillo $\mathrm{R}$ : Long-term histopathologic findings in two cerebral aneurysms embolized with Guglielmi detachable coils. AJNR Am J Neuroradiol 20:549-552, 1999

12. Chalouhi N, Polifka A, Daou B, Kung D, Barros G, Tjoumakaris $S$, et al: In-pipeline stenosis: incidence, predictors, and clinical outcomes. Neurosurgery 77:875-879, 2015

13. Chalouhi N, Tjoumakaris S, Dumont AS, Gonzalez LF, Randazzo C, Starke RM, et al: Treatment of posterior circulation 
aneurysms with the Pipeline embolization device. Neurosurgery 72:883-889, 2013

14. Daou B, Starke RM, Chalouhi N, Barros G, Tjoumakaris S, Rosenwasser RH, et al: P2Y12 reaction units: effect on hemorrhagic and thromboembolic complications in patients with cerebral aneurysms treated with the Pipeline embolization device. Neurosurgery 78:27-33, 2016

15. Delgado Almandoz JE, Crandall BM, Scholz JM, Fease JL, Anderson RE, Kadkhodayan Y, et al: Last-recorded P2Y12 reaction units value is strongly associated with thromboembolic and hemorrhagic complications occurring up to 6 months after treatment in patients with cerebral aneurysms treated with the Pipeline Embolization Device. AJNR Am J Neuroradiol 35:128-135, 2014

16. Delgado Almandoz JE, Crandall BM, Scholz JM, Fease JL, Anderson RE, Kadkhodayan Y, et al: Pre-procedure P2Y12 reaction units value predicts perioperative thromboembolic and hemorrhagic complications in patients with cerebral aneurysms treated with the Pipeline Embolization Device. J Neurointerv Surg 5 (Suppl 3):iii3-iii10, 2013

17. Drazin D, Choulakian A, Nuño M, Kornbluth P, Alexander MJ: Body weight: a risk factor for subtherapeutic antithrombotic therapy in neurovascular stenting. J Neurointerv Surg 3:177-181, 2011

18. Dumont TM, Kan P, Snyder KV, Hopkins LN, Siddiqui AH, Levy EI: Adjunctive use of eptifibatide for complication management during elective neuroendovascular procedures. J Neurointerv Surg 5:226-230, 2013

19. Faught RW, Satti SR, Hurst RW, Pukenas BA, Smith MJ: Heterogeneous practice patterns regarding antiplatelet medications for neuroendovascular stenting in the USA: a multicenter survey. J Neurointerv Surg 6:774-779, 2014

20. Fifi JT, Brockington C, Narang J, Leesch W, Ewing SL, Bennet $\mathrm{H}$, et al: Clopidogrel resistance is associated with thromboembolic complications in patients undergoing neurovascular stenting. AJNR Am J Neuroradiol 34:716-720, 2013

21. Gaglia MA, Torguson R, Pakala R, Xue Z, Sardi G, Suddath WO, et al: Correlation between light transmission aggregometry, VerifyNow P2Y12, and VASP-P platelet reactivity assays following percutaneous coronary intervention. $\mathbf{J}$ Interv Cardiol 24:529-534, 2011

22. Goh C, Churilov L, Mitchell P, Dowling R, Yan B: Clopidogrel hyper-response and bleeding risk in neurointerventional procedures. AJNR Am J Neuroradiol 34:721-726, 2013

23. Gralla J, Rennie AT, Corkill RA, Lalloo ST, Molyneux A, Byrne JV, et al: Abciximab for thrombolysis during intracranial aneurysm coiling. Neuroradiology 50:1041-1047, 2008

24. Gurbel PA, Bliden KP, Butler K, Antonino MJ, Wei C, Teng $\mathrm{R}$, et al: Response to ticagrelor in clopidogrel nonresponders and responders and effect of switching therapies: the RESPOND study. Circulation 121:1188-1199, 2010

25. Hanel RA, Taussky P, Dixon T, Miller DA, Sapin M, Nordeen JD, et al: Safety and efficacy of ticagrelor for neuroendovascular procedures. A single center initial experience. J Neurointerv Surg 6:320-322, 2014

26. Heller RS, Dandamudi V, Lanfranchi M, Malek AM: Effect of antiplatelet therapy on thromboembolism after flow diversion with the Pipeline Embolization Device. J Neurosurg 119:1603-1610, 2013

27. Holmes DR Jr, Dehmer GJ, Kaul S, Leifer D, O'Gara PT, Stein CM: ACCF/AHA clopidogrel clinical alert: approaches to the FDA "boxed warning": a report of the American College of Cardiology Foundation Task Force on clinical expert consensus documents and the American Heart Association endorsed by the Society for Cardiovascular Angiography and Interventions and the Society of Thoracic Surgeons. J Am Coll Cardiol 56:321-341, 2010

28. Hwang G, Huh W, Lee JS, Villavicencio JB, Villamor RB Jr, Ahn SY, et al: Standard vs modified antiplatelet preparation for preventing thromboembolic events in patients with high on-treatment platelet reactivity undergoing coil embolization for an unruptured intracranial aneurysm: a randomized clinical trial. JAMA Neurol 72:764-772, 2015

29. Jeong HW, Jin SC: Intra-arterial infusion of a glycoprotein IIb/IIIa antagonist for the treatment of thromboembolism during coil embolization of intracranial aneurysm: a comparison of abciximab and tirofiban. AJNR Am J Neuroradiol 34:1621-1625, 2013

30. Johnson JA, Cavallari LH: Pharmacogenetics and cardiovascular disease-implications for personalized medicine. Pharmacol Rev 65:987-1009, 2013

31. Jones RG, Davagnanam I, Colley S, West RJ, Yates DA: Abciximab for treatment of thromboembolic complications during endovascular coiling of intracranial aneurysms. AJNR Am J Neuroradiol 29:1925-1929, 2008

32. Kan P, Siddiqui AH, Veznedaroglu E, Liebman KM, Binning MJ, Dumont TM, et al: Early postmarket results after treatment of intracranial aneurysms with the Pipeline embolization device: a U.S. multicenter experience. Neurosurgery 71:1080-1088, 2012

33. Kang HS, Kwon BJ, Kim JE, Han MH: Preinterventional clopidogrel response variability for coil embolization of intracranial aneurysms: clinical implications. AJNR Am J Neuroradiol 31:1206-1210, 2010

34. Kashiwazaki D, Kuwayama N, Akioka N, Hayakawa Y, Kuroda S: The roles and issues of P2Y12 percent inhibition assessed by VerifyNow assay for patients undergoing neurointervention: a prospective study. J Stroke Cerebrovasc Dis 23:1830-1836, 2014

35. Koerner H, Derveaux C, Alexandrou M, Graeber S, Roth C, Papanagiotou $\mathrm{P}$, et al: Do clopidogrel nonresponders have an increased risk of adverse events during supra-aortal angioplasty and stenting? Stroke Res Treat 2012:904534, 2012

36. Lee DH, Arat A, Morsi H, Shaltoni H, Harris JR, Mawad ME: Dual antiplatelet therapy monitoring for neurointerventional procedures using a point-of-care platelet function test: a single-center experience. AJNR Am J Neuroradiol 29:1389-1394, 2008

37. Levitt MR, Moon K, Albuquerque FC, Mulholland CB, Kalani MY, McDougall CG: Intraprocedural abciximab bolus versus pretreatment oral dual antiplatelet medication for endovascular stenting of unruptured intracranial aneurysms. J Neurointerv Surg 8:909-912, 2016

38. Lin N, Lanzino G, Lopes DK, Arthur AS, Ogilvy CS, Ecker $\mathrm{RD}$, et al: Treatment of distal anterior circulation aneurysms with the Pipeline embolization device: a US multicenter experience. Neurosurgery 79:14-22, 2016

39. Mahaffey KW, Wojdyla DM, Carroll K, Becker RC, Storey RF, Angiolillo DJ, et al: Ticagrelor compared with clopidogrel by geographic region in the Platelet Inhibition and Patient Outcomes (PLATO) trial. Circulation 124:544-554, 2011

40. Mandava P, Thiagarajan P, Kent TA: Glycoprotein IIb/IIIa antagonists in acute ischaemic stroke: current status and future directions. Drugs 68:1019-1028, 2008

41. Michelson AD: Methods for the measurement of platelet function. Am J Cardiol 103 (3 Suppl):20A-26A, 2009

42. Mocco J, Fargen KM, Albuquerque FC, Bendok BR, Boulos AS, Carpenter JS, et al: Delayed thrombosis or stenosis following Enterprise-assisted stent-coiling: is it safe? Midterm results of the interstate collaboration of Enterprise stent coiling. Neurosurgery 69:908-914, 2011

43. Müller-Schunk S, Linn J, Peters N, Spannagl M, Deisenberg M, Brückmann H, et al: Monitoring of clopidogrel-related platelet inhibition: correlation of nonresponse with clinical outcome in supra-aortic stenting. AJNR Am J Neuroradiol 29:786-791, 2008

44. Nishi H, Nakahara I, Matsumoto S, Hashimoto T, Ohta T, 
Sadamasa N, et al: Platelet reactivity and hemorrhage risk in neurointerventional procedures under dual antiplatelet therapy. J Neurointerv Surg 8:949-953, 2016

45. Nordeen JD, Patel AV, Darracott RM, Johns GS, Taussky P, Tawk RG, et al: Clopidogrel resistance by P2Y12 platelet function testing in patients undergoing neuroendovascular procedures: incidence of ischemic and hemorrhagic complications. J Vasc Interv Neurol 6:26-34, 2013

46. Oran I, Cinar C, Bozkaya H, Korkmaz M: Tailoring platelet inhibition according to multiple electrode aggregometry decreases the rate of thrombotic complications after intracranial flow-diverting stent implantation. J Neurointerv Surg 7:357-362, 2015

47. Pandya DJ, Fitzsimmons BFM, Wolfe TJ, Hussain SI, Lynch JR, Ortega-Gutierrez S, et al: Measurement of antiplatelet inhibition during neurointerventional procedures: the effect of antithrombotic duration and loading dose. J Neuroimaging 20:64-69, 2010

48. Paniccia R, Priora R, Liotta AA, Abbate R: Platelet function tests: a comparative review. Vasc Health Risk Manag 11:133-148, 2015

49. Paré G, Mehta SR, Yusuf S, Anand SS, Connolly SJ, Hirsh J, et al: Effects of CYP2C19 genotype on outcomes of clopidogrel treatment. N Engl J Med 363:1704-1714, 2010

50. Park JH, Kim JE, Sheen SH, Jung CK, Kwon BJ, Kwon OK, et al: Intraarterial abciximab for treatment of thromboembolism during coil embolization of intracranial aneurysms: outcome and fatal hemorrhagic complications. J Neurosurg 108:450-457, 2008

51. Parodi G, Bellandi B, Venditti F, Carrabba N, Valenti R, Migliorini A, et al: Residual platelet reactivity, bleedings, and adherence to treatment in patients having coronary stent implantation treated with prasugrel. Am J Cardiol 109:214218, 2012

52. Pierot L, Cognard C, Anxionnat R, Ricolfi F: Ruptured intracranial aneurysms: factors affecting the rate and outcome of endovascular treatment complications in a series of 782 patients (CLARITY study). Radiology 256:916-923, 2010

53. Prabhakaran S, Wells KR, Lee VH, Flaherty CA, Lopes DK: Prevalence and risk factors for aspirin and clopidogrel resistance in cerebrovascular stenting. AJNR Am J Neuroradiol 29:281-285, 2008

54. Price MJ: Bedside evaluation of thienopyridine antiplatelet therapy. Circulation 119:2625-2632, 2009

55. Price MJ, Angiolillo DJ, Teirstein PS, Lillie E, Manoukian SV, Berger PB, et al: Platelet reactivity and cardiovascular outcomes after percutaneous coronary intervention: a timedependent analysis of the Gauging Responsiveness with a VerifyNow P2Y12 assay: Impact on Thrombosis and Safety (GRAVITAS) trial. Circulation 124:1132-1137, 2011

56. Price MJ, Berger PB, Teirstein PS, Tanguay JF, Angiolillo DJ, Spriggs D, et al: Standard- vs high-dose clopidogrel based on platelet function testing after percutaneous coronary intervention: the GRAVITAS randomized trial. JAMA 305:1097-1105, 2011 (Erratum in JAMA 305:2174, 2011)

57. Ramakrishnan P, Yoo AJ, Rabinov JD, Ogilvy CS, Hirsch JA, Nogueira RG: Intra-arterial eptifibatide in the management of thromboembolism during endovascular treatment of intracranial aneurysms: case series and a review of the literature. Interv Neurol 2:19-29, 2013

58. Ries T, Siemonsen S, Grzyska U, Zeumer H, Fiehler J: Abciximab is a safe rescue therapy in thromboembolic events complicating cerebral aneurysm coil embolization: single center experience in 42 cases and review of the literature. Stroke 40:1750-1757, 2009

59. Scott SA, Sangkuhl K, Gardner EE, Stein CM, Hulot JS, Johnson JA, et al: Clinical Pharmacogenetics Implementa- tion Consortium guidelines for cytochrome P450-2C19 (CYP2C19) genotype and clopidogrel therapy. Clin Pharmacol Ther 90:328-332, 2011

60. Scott SA, Sangkuhl K, Shuldiner AR, Hulot JS, Thorn CF, Altman RB, et al: PharmGKB summary: very important pharmacogene information for cytochrome P450, family 2 , subfamily C, polypeptide 19 . Pharmacogenet Genomics 22:159-165, 2012

61. Scott SA, Sangkuhl K, Stein CM, Hulot JS, Mega JL, Roden DM, et al: Clinical Pharmacogenetics Implementation Consortium guidelines for CYP2C19 genotype and clopidogrel therapy: 2013 update. Clin Pharmacol Ther 94:317-323, 2013

62. Sedat J, Chau Y, Gaudard J, Suissa L, Lachaud S, Lonjon M: Administration of eptifibatide during endovascular treatment of ruptured cerebral aneurysms reduces the rate of thromboembolic events. Neuroradiology 57:197-203, 2015

63. Sibbing D, Byrne RA, Bernlochner I, Kastrati A: High platelet reactivity and clinical outcome-fact and fiction. Thromb Haemost 106:191-202, 2011

64. Skillman KL, Caruthers RL, Johnson CE: Stability of an extemporaneously prepared clopidogrel oral suspension. Am J Health Syst Pharm 67:559-561, 2010

65. Starnes HB, Patel AA, Stouffer GA: Optimal use of platelet glycoprotein IIb/IIIa receptor antagonists in patients undergoing percutaneous coronary interventions. Drugs 71:20092030, 2011

66. Stetler WR, Chaudhary N, Thompson BG, Gemmete JJ, Maher CO, Pandey AS: Prasugrel is effective and safe for neurointerventional procedures. J Neurointerv Surg 5:332-336, 2013

67. Tan LA, Keigher KM, Munich SA, Moftakhar R, Lopes DK: Thromboembolic complications with Pipeline Embolization Device placement: impact of procedure time, number of stents and pre-procedure P2Y12 reaction unit (PRU) value. J Neurointerv Surg 7:217-221, 2015

68. Tantry US, Bonello L, Aradi D, Price MJ, Jeong YH, Angiolillo DJ, et al: Consensus and update on the definition of on-treatment platelet reactivity to adenosine diphosphate associated with ischemia and bleeding. J Am Coll Cardiol 62:2261-2273, 2013

69. van Werkum JW, Harmsze AM, Elsenberg EH, Bouman HJ, ten Berg JM, Hackeng CM: The use of the VerifyNow system to monitor antiplatelet therapy: a review of the current evidence. Platelets 19:479-488, 2008

70. Wong P, Tesoro E, Aletich V, Alaraj A: Accumetrics-based clopidogrel dosing in endovascular neurosurgery. Neurol Res 37:998-1005, 2015

\section{Disclosures}

The authors report no conflict of interest concerning the materials or methods used in this study or the findings specified in this paper.

\section{Author Contributions}

Conception and design: Cook, Kim, Fraser. Drafting the article: all authors. Critically revising the article: all authors. Reviewed submitted version of manuscript: all authors. Approved the final version of the manuscript on behalf of all authors: Cook.

\section{Correspondence}

Aaron M. Cook, UK Healthcare, 800 Rose St., H110, Lexington, KY 40536. email: amcook0@email.uky.edu. 